Peter Gerlinger, Yann Hendrik Simsont, Numerical investigation of scramjet strut injector cooling for different fuel mass fluxes and strut material properties at Mach 8 flight conditions, Acta Astronautica 160 (2019) 353-364.

The original publication is available at www.elsevier.com

https://www.sciencedirect.com/science/article/pii/S0094576518321283?via\%3Dihub

http://dx.doi.org/10.1016/j.actaastro.2019.04.042

(C) <year>. This manuscript version is made available under the CC-BY-NC-ND 4.0 license http://creativecommons.org/licenses/by-nc-nd/4.0/ 


\title{
Numerical investigation of scramjet strut injector cooling for different fuel mass fluxes and strut material properties at Mach 8 flight conditions
}

\author{
Peter Gerlinger*, Yann Hendrik Simsont \\ Institute of Combustion Technology of Aerospace Engineering, University of Stuttgart, \\ Pfaffenwaldring 38-40, 70569 Stuttgart, Germany
}

\begin{abstract}
The thermal load on a lobed scramjet (supersonic combustion ramjet) strut injector is investigated numerically for Mach 8 flight conditions. For this purpose, coupled 3D RANS (Reynolds Averaged Navier-Stokes) simulations of the supersonic external flow field around the strut injector and the subsonic strut internal hydrogen flow are performed. The temperature distribution in the solid strut material is simulated by a separate code, which exchanges data with the fluid solvers. Due to the predominantly low Mach numbers inside the strut, an all-Mach number preconditioning is required to enable convergence of the compressible flow solver. The air flow Mach number in the combustion chamber is three. Different strut materials (copper, silicone carbide, molybdenum, and tungsten) with different heat capacities and thermal conductivities are compared. Moreover, the impact of the height of the blunt tip of the strut on the heat load is investigated. Due to a small detached normal shock wave upstream of the strut, the temperatures at the tip become very high; this part is notably difficult to cool. In order to keep the strut temperatures acceptable, it is cooled actively by the hydrogen that is injected for combustion. A sophisticated internal strut structure is needed to direct the hydrogen towards regions of highest heat load. Different strut geometries, fuel mass fluxes (equivalence ratios), and strut materials are investigated.
\end{abstract}

\footnotetext{
${ }^{*}$ Corresponding author

Email address: peter.gerlinger@dlr.de (Peter Gerlinger)
} 
Keywords: Scramjet, strut injector, conjugate heat transfer, strut cooling

\section{Introduction}

The cooling of scramjet engines is an important issue for practical applications. Due to the high total temperatures of hypersonic flows, this is a complicated task. The most extreme heat loads in a scramjet usually appear in the combustion chamber. Here, the incoming air already is compressed to a level which enables combustion, and the flow velocity is relatively low compared to the flight velocity. Moreover, there is heat release due to combustion. Similar to rocket engines, the injected fuel is the best choice of coolant. Otherwise, an additional cooling fluid with corresponding tanks and supply systems is needed, which must increase the weight. Thus the question arises, whether there is enough fuel to cool all relevant parts (combustor walls, fuel injectors, and fractions of inlet and nozzle) at continuous operation. In case of flame stabilization by cavities [1] or in case of wall fuel injection [2] with flames close to solid walls, the nearby material is exposed to the highest temperatures. Regenerative, film, and transpiration cooling techniques are under investigation $[3,4,5,6]$. Additionally, cryogenic fuels such as liquid hydrogen or methane can be chosen, which, similar to rocket engines, offer good cooling properties before they are burned. Another possibility is hydrocarbon fuels (e.g. n-octane [7], n-decane [8, 9], n-dodecane [10]), which, due to endothermic chemical cracking (e.g. in the cooling channels [11]), can work as a heat sink [7] under certain conditions. A second advantage of this procedure is, that after cracking, the products often have shorter ignition delay times (e.g. due the production of hydrogen). Nevertheless, the cooling capability of the fuel is limited, especially at high flight Mach numbers [4].

With respect to strut cooling, it is important to note that after the combustion chamber and/or inlet cooling, the fuel entering the strut may be relatively hot. Moreover, the hydrogen mass flux is basically defined by the required thrust (which additionally may change over time). If combustion is not negatively affected, an increase in fuel mass flux above a stoichiometric ratio, in order to improve strut cooling is possible. In contrast to stoichiometric conditions, where at complete combustion water is the only product, the consequence now is unburned hydrogen in the exhaust gas. As this excess fuel must be stored and transported, it has to be avoided. Thus a scramjet 
strut injector should be designed in such a way (material, geometry, design of internal flow) that sufficient cooling of the strut for the complete range of operation is ensured for the fuel mass flux, which is defined by thrust requirements. If needed, an additional method for regulation could be to modify the strut fuel inflow temperature by mixing fresh cold fuel from the tanks with fuel, that is already used for engine cooling. With these measures, it has to be kept in mind that the degree of cooling changes fuel injection temperature, pressure, and velocity. These parameters are important for ignition delay, mixing, and combustion and should not be affected adversely. Due to these complex interactions, it is important to look for the best possible cooling strategy for a chosen strut geometry in order to reduce the requirements elsewhere.

One possible concept for scramjet engine fuel supply is the use of strut injectors located on the channel axis $[12,13,14,15,16,17,18]$. This way, the fuel is directly injected into the core of the air stream and it is possible to achieve fast and efficient mixing [18]. Moreover, under ideal conditions, the flame is positioned downstream of the strut and causes no additional heat load either for the strut or the walls $[9,12,13]$. Struts which are exposed to flames of upstream fuel injectors should be avoided, at least at higher flight Mach numbers. For example, staged combustion concepts with perpendicularly installed strut injectors [19] can improve mixing, but the second strut is subjected to even higher temperatures than the first one. Without efficient cooling, this can damage the strut at continuous operation. Alternatively, two [17] or more [20] struts can be used in parallel at the same axial position or only slightly shifted from each other. This improves mixing without causing additional heat load for the strut. Disadvantages of this technique are an increased blockage of the main flow and unfavorable strut-strut shock wave interactions.

The following simulations are based on the assumption that combustion takes place downstream of the strut. This allows for conjugate heat transfer strut injector studies to be carried out without taking combustion into account. In order to achieve fast mixing (short combustor length), a lobed strut structure which has proven to enable rapid mixing due to creation of streamwise vorticity [12] is used. A disadvantage of struts compared to wall injectors, however, is the cooling challenge; this is the topic of the present paper. As the strut is located at the center of the channel, it is exposed to the incoming high speed flow. In the investigated test case with Mach 8 flight conditions, the static temperature inside the combustor is approxi- 
mately $1160 \mathrm{~K}$, which corresponds to a total temperature of about $3250 \mathrm{~K}$. Thus, the tip of the strut in particular may suffer from very high heat loads. Moreover, fuel is required for active cooling which may never be switched off completely. The required minimum fuel mass flow is an important parameter for a given set of operating conditions.

A large number of different strut injector concepts has been proposed in literature, e.g. $[21,18,22,23,17]$. Most of them use streamwise vortices to improve mixing. These struts are usually investigated or optimized (either experimentally or numerically) with respect to mixing efficiency [18, 24], total pressure loss [14] or other performance parameters [25]. However, the thermal load on the strut is usually not considered. Many of the proposed concepts may not be used under real high speed conditions because they can not be cooled efficiently. For example, a sharp tip is difficult to cool and may burn down at high speed conditions, as will be shown later. Another aspect for practical application is a suited structural design of the scramjet combustor including fuel injectors. There are not many papers dealing with this subject and thermally induced stresses [26, 27, 28, 29], which is not treated in the present paper. Instead the focus is on the heat load of the strut.

To approximate the wall heat flux densities (heat flux per unit area) at the Mach 8 flight and Mach 3 combustor entrance conditions studied in this paper (see Table 1), flat plate boundary layers (for air) with different constant wall temperatures are calculated. The corresponding wall heat flux densities after $1 \mathrm{~m}$ plate length strongly depend on the chosen wall temperature, as can be seen in Fig. 1. If the wall temperature could be increased from 700 $\mathrm{K}$ to $1900 \mathrm{~K}$, the wall heat flux densitiy would decrease by $70 \%$. Thus, less coolant is required if the combustor walls are able to sustain higher temperatures. This could be an advantage of ceramic composite materials. A lower heat conductivity may be compensated by higher possible maximum temperatures. Additionally, the material should have good emissivity.

There are a number of papers in literature, where cooling concepts of complete scramjet engines are investigated in simplified ways $[3,30,31,32,8]$. However, only a few studies currently exist which investigate cooling concepts for parts of a scramjet combustor (e.g. wall structures, cooling channels [11], or injectors $[12,13,16,9])$ by detailed 3D numerical simulations. This paper treats such conjugate heat transfer problems numerically. The investigated lobed strut geometry was first presented in [33] and geometrical variants of it have been tested e.g. in $[18,34,15]$. A first simulation with heat transfer for a copper strut at real flight conditions followed, but only for one equivalence 


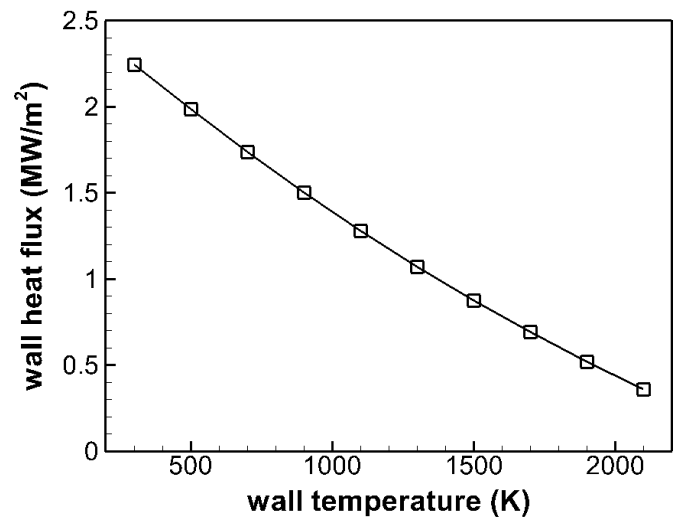

Figure 1: Calculated wall heat flux densities of a Mach 3 turbulent boundary layer (Mach 8 flight conditions) using different constant wall temperatures.

ratio $(\Phi=0.7)[12,13]$. Experimental and numerical studies of a similar strut have since been performed at laboratory conditions with a total temperature of $1300 \mathrm{~K}$ [16]. The present paper studies (again for Mach 8 flight conditions with a total temperature of $3250 \mathrm{~K}$ ):

1. The impact of the geometrical choice of the tip of the strut on its thermal load.

2. The influence of different hydrogen mass fluxes on cooling.

3. The effect of different strut materials on heat fluxes and peak temperatures.

To this end, a large number of relatively complex simulations is performed which give insight into the fluid dynamical processes as well as in the temperature distributions in the strut injector. Due to the large variety of possible strut materials and thus material properties, it is not the scope of this paper to recommend a certain material for a strut. Instead the authors want to show the impact of a given thermal conductivity, density, and specific heat of a material on the heat fluxes, maximum temperatures in the strut, and the required mass fluxes of coolant. To the authors knowledge this is the first paper where such a comprehensive study of different impact parameters on the thermal behavior of a strut injector has been performed. 


\section{Numerical solvers}

For the strut injector problem described above, a coupled solution of the gas-phase flows inside and outside the strut together with the heat conduction in the solid material is required. This is achieved by two separate codes.

\section{Gas phase}

All simulations in this paper are based on the following set of averaged, compressible, non-reactive equations for mass, momentum, energy, and species:

$$
\begin{gathered}
\frac{\partial \bar{\rho}}{\partial t}+\frac{\partial}{\partial x_{i}}\left(\bar{\rho} \tilde{u}_{i}\right)=0 \\
\frac{\partial}{\partial t}\left(\bar{\rho} \tilde{u}_{i}\right)+\frac{\partial}{\partial x_{j}}\left(\bar{\rho} \tilde{u}_{i} \tilde{u}_{j}\right)=\frac{\partial \tau_{i j}^{\mathrm{eff}}}{\partial x_{j}}-\frac{\partial \bar{p}}{\partial x_{i}}, \\
\frac{\partial}{\partial t}(\bar{\rho} \tilde{E})+\frac{\partial}{\partial x_{j}}\left(\bar{\rho} \tilde{u}_{j} \tilde{H}\right)=\frac{\partial}{\partial x_{j}}\left(\tilde{u}_{i} \tau_{i j}^{\mathrm{eff}}-q_{j}^{\mathrm{eff}}\right), \\
\frac{\partial}{\partial t}\left(\bar{\rho} \tilde{Y}_{\alpha}\right)+\frac{\partial}{\partial x_{j}}\left(\bar{\rho} \tilde{u}_{j} \tilde{Y}_{\alpha}\right)=\frac{\partial j_{\alpha j}^{\mathrm{eff}}}{\partial x_{j}}
\end{gathered}
$$

where the elements of the effective stress tensor

$$
\tau_{i j}^{\mathrm{eff}}=2 \mu^{\mathrm{eff}}\left[\frac{1}{2}\left(\frac{\partial \tilde{u}_{i}}{\partial x_{j}}+\frac{\partial \tilde{u}_{j}}{\partial x_{i}}\right)-\frac{1}{3} \frac{\partial \tilde{u}_{k}}{\partial x_{k}}\right]-\frac{2}{3} \rho k \delta_{i j}
$$

are calculated from molecular $(m)$ and eddy viscosity $(t)$ contributions by $\mu^{\text {eff }}=\mu^{\mathrm{m}}+\mu^{\mathrm{t}}$. Similarely, effective energy

$$
q_{j}^{\mathrm{eff}}=-\lambda^{\mathrm{eff}} \frac{\partial \tilde{T}}{\partial x_{j}}-\mu^{\mathrm{eff}} \frac{\partial k}{\partial x_{j}}+\sum_{\alpha=1}^{N_{k}} h_{\alpha} j_{\alpha}^{\mathrm{eff}}
$$

and species fluxes

$$
j_{\alpha j}^{\mathrm{eff}}=-\bar{\rho} D_{\alpha}^{\mathrm{eff}} \frac{\partial \tilde{Y}_{\alpha}}{\partial x_{j}}
$$

are calculated, which use $\lambda^{\text {eff }}=\lambda^{\mathrm{m}}+\lambda^{\mathrm{t}}$ and $D_{\alpha}^{\mathrm{eff}}=D_{\alpha}^{\mathrm{m}}+D_{\alpha}^{\mathrm{t}}$ for the thermal conductivity and diffusion coefficients, respectively. In the equations above, $\rho$ is the density, $p$ the pressure, $u_{i}$ the velocity component in $i$-coordinate direction, $E=e+0.5 u_{i}^{2}+q^{2}$ is the total energy, $e$ the specific internal 
energy, $H$ the total enthalpy, and $Y_{\alpha}$ the mass fraction of species $\alpha$. Further, $t$ is the time, $x_{i}$ are the coordinate directions, and ${ }^{-}$and ${ }^{\sim}$ indicate Reynolds and Favre averages, respectively. In this paper, Einstein summation is taken over the indices $i, j$, and $k$, but not over the $N_{k}$ species $\alpha$. For turbulence closure the low-Reynolds number $q-\omega$ model from Coakley and Huang [35]

$$
\begin{gathered}
\frac{\partial}{\partial t}(\bar{\rho} q)+\frac{\partial}{\partial x_{j}}\left(\bar{\rho} \tilde{u}_{j} q\right)=\frac{\partial}{\partial x_{j}}\left[\left(\mu^{m}+\frac{\mu^{t}}{\sigma_{q}}\right) \frac{\partial q}{\partial x_{j}}\right]+S_{q} \\
\frac{\partial}{\partial t}(\bar{\rho} \omega)+\frac{\partial}{\partial x_{j}}\left(\bar{\rho} \tilde{u}_{j} \omega\right)=\frac{\partial}{\partial x_{j}}\left[\left(\mu^{m}+\frac{\mu^{t}}{\sigma_{\omega}}\right) \frac{\partial \omega}{\partial x_{j}}\right]+S_{\omega}
\end{gathered}
$$

is chosen, which has proven to be reliable and stable for scramjet applications $[33,18]$. The model uses the turbulence variables $q=\sqrt{k}$ ( $k$ is the turbulent kinetic energy) and $\omega$, which is a turbulent frequency. Details concerning the source terms $S_{q}$ and $S_{\omega}$ as well as all constants (e.g. $\sigma_{q}$ and $\sigma_{\omega}$ ) may be found in the original literature [35]. The combined set of equations given above may be expressed by

$$
\frac{\partial \mathbf{Q}}{\partial t}+\frac{\partial\left(\mathbf{F}_{j}-\mathbf{F}_{\nu, j}\right)}{\partial x_{j}}=\mathbf{S}
$$

where $\mathbf{Q}=\left[\bar{\rho}, \bar{\rho} \widetilde{u}_{i}, \bar{\rho} \widetilde{E}, \bar{\rho} q, \bar{\rho} \omega, \bar{\rho} \widetilde{Y}_{\alpha}\right]^{t}$ is the conservative variable vector and $\mathbf{F}$ and $\mathbf{F}_{\nu}$ are inviscid and viscous flux vectors. In the present test cases, there is a large disparity in flow Mach number between the internal low speed flow (which dominates large parts inside the strut) and the acceleration in the nozzle up to the (strut external) combustor Mach number of 3. An all-Mach number preconditioning $[36,13]$ was required to achieve convergence of the compressible flow solver. For the steady-state solutions in this paper, no dual time stepping is required and the preconditioned version of Eq. (10)

$$
\boldsymbol{\Gamma} \frac{\partial \mathbf{Q}_{p}}{\partial \tau}+\frac{\partial\left(\mathbf{F}_{j}-\mathbf{F}_{\nu, j}\right)}{\partial x_{j}}=\mathbf{S}
$$

is solved, where $\mathbf{Q}_{\mathrm{p}}=\left[\bar{p}, \widetilde{u}_{i}, \widetilde{T}, q, \omega, \widetilde{Y}_{\alpha}\right]^{t}$ is the primitive variable vector and $\tau$ is a pseudo time. The preconditioning matrix $\boldsymbol{\Gamma}$ is presented in [13] and is based on those of Choi and Merkle [36]. To solve the preconditioned set of equations, the in-house code TASCOM3D [37, 38, 39] is used. TASCOM3D has been developed for high speed flows with and without combustion over 
more than two decades. The unsteady form of governing equations is integrated in time using an implicit finite-volume Lower-Upper Symmetric GaußSeidel (LU-SGS) algorithm [40, 41]. Alongside the inviscid Jacobians, simplified viscous Jacobians (based on the thin-layer Navier-Stokes equations) are included in the implicit part. In Eq. (12) this is shown for the $j$-direction only. The discretized implicit LU-SGS scheme is given by $[41,37]$

$$
\begin{aligned}
& {\left[\boldsymbol{\Gamma}+\Delta \tau\left(\tilde{\mathbf{A}}_{i, j, k}^{+}-\tilde{\mathbf{A}}_{i, j, k}^{-}+\tilde{\mathbf{B}}_{i, j, k}^{+}-\tilde{\mathbf{B}}_{i, j, k}^{-}+\tilde{\mathbf{C}}_{i, j, k}^{+}-\tilde{\mathbf{C}}_{i, j, k}^{-}+2 \tilde{\mathbf{T}}_{i, j, k}-\tilde{\mathbf{H}}_{i, j, k}\right)\right.} \\
& -\Delta \tau\left(\tilde{\mathbf{A}}_{i-1, j, k}^{+}+\tilde{\mathbf{B}}_{i, j-1, k}^{+}+\tilde{\mathbf{C}}_{i, j, k-1}^{+}+\tilde{\mathbf{T}}_{i, j-1, k}\right) \\
& \left.-\Delta \tau\left(\tilde{\mathbf{A}}_{i+1, j, k}^{-}+\tilde{\mathbf{B}}_{i, j+1, k}^{-}+\tilde{\mathbf{C}}_{i, j, k+1}^{-}+\tilde{\mathbf{T}}_{i, j+1, k}\right)\right] \Delta \mathbf{Q}_{p, i, j, k}^{n+1} \\
& =-\Delta \tau \mathbf{R}_{i, j, k}^{n}
\end{aligned}
$$

where $\tilde{\mathbf{A}}, \tilde{\mathbf{B}}$, and $\tilde{\mathbf{C}}$ are Jacobians resulting from linearization of the corresponding inviscid flux vectors in the three coordinate directions. To ensure diagonal dominance, the upwind differenced Jacobians on the cell interfaces $\tilde{\mathbf{A}}, \tilde{\mathbf{B}}$, and $\tilde{\mathbf{C}}$ are split in + and - matrices containing only positive or negative eigenvalues $[40,41]$. $\mathbf{T}$ are centrally differenced Jacobians of the viscous fluxes and $\mathbf{H}=d \mathbf{S} / d \mathbf{Q}$ is the source Jacobian due to chemistry (not used in this paper) and turbulence $[41,37$. The turbulence equations are solved in a loosely coupled form with the fluid motion. Finally, $\mathbf{R}$ is the discretized residual and $n$ the iteration index. If the diagonal, lower, and upper Jacobians of Eq. (12) are combined to form the operators $D, L$, and $U$, Eq. (12) can be expressed by

$$
(D+L+U) \Delta \mathbf{Q}_{p}^{n+1}=-\Delta \tau \mathbf{R}^{n} .
$$

Approximately factored this equation is solved in two steps [41].

Lower sweep:

$$
(D+L) \Delta \overline{\mathbf{Q}}_{p}=-\Delta \tau \mathbf{R}^{n},
$$

Upper sweep:

$$
(D+U) \Delta \mathbf{Q}_{p}^{n+1}=D \Delta \overline{\mathbf{Q}}_{p}
$$

and the solution is updated by $\mathbf{Q}_{p}^{n+1}=\mathrm{Q}_{p}^{n}+\Delta \mathbf{Q}_{p}^{n+1}$. The accuracy of TASCOM3D is up to sixth order in space and third order in time [39]. However, the steady-state simulations presented in this paper are calculated with a second order discretization in space. In contrast to time accurate unsteady 
flows, this has proven to be sufficient for steady-state RANS simulations [39]. For the interface flux calculation, the $\mathrm{AUSM}^{+}$-up flux vector splitting [42] is used. This method works well even at very low Mach numbers. The calculation of the viscous fluxes is realized by a second order central discretization. The high numerical stability of the implicit LU-SGS scheme is advantageous for the following simulations, which are not only distinguished by large differences in Mach number but also by very fine high aspect ratio grids close to solid walls, shock waves, and a complex strut internal flow field. A three-component gas mixture (nitrogen, oxygen, hydrogen) is considered. TASCOM3D is fully vectorized and parallelized using MPI and is based on structured multi-block grids. Both the turbulent Prandtl and Schmidt numbers are assumed to be 0.7. The code has been validated over many years, especially for high speed flows and supersonic combustion, using a large number of test cases. Some examples may be found in [12, 18, 43, 33, 34, 37, 38, 39]. Moreover, validations have been performed for an identical strut at lower temperatures and Mach numbers, which could be realized at a ground test facility $[18,33,34]$. Based on these validations, the code is used in this paper to investigate the strut behavior at Mach 8 flight conditions. At ground test facilities in continuous operation, correspondingly high temperatures may not be realized. In [44], $2450 \mathrm{~K}$ total temperature is reached by precombustion, which is still significantly lower than the $3250 \mathrm{~K}$ used in the present study. On the other hand, high enthalpy shock tunnels which are able to achieve such high total temperatures, only enable a realization over extremely short periods, much too short to investigate a heating of the strut. For these reasons numerical simulations or flight experiments seem to be the only possibility to investigate struts under realistic conditions.

\section{Solid phase}

To calculate the temperature distribution inside the strut, the threedimensional energy equation in a temperature formulation

$$
\rho V c_{p} \frac{\partial T}{\partial t}+\oint_{S} \lambda \nabla T d S=0
$$

is solved, where the heat fluxes are calculated from Fourier's law. The properties of the strut material are the constant density $\rho$, the temperature dependent specific heat $c_{p}(T)$, and the thermal conductivity $\lambda(T) . V$ is the 
volume and $S$ the surface of a computational cell. The thermal diffusivity $a(T)$ of the material is calculated from

$$
a(T)=\frac{\lambda(T)}{\rho c_{p}(T)} .
$$

At the interface between gas and solid, a constant heat flux density is assumed and the wall temperature $T_{\text {wall }}$ is obtained from

$$
\lambda_{g} \frac{T_{g}-T_{\text {wall }}}{\Delta y_{g}}=\lambda_{s} \frac{T_{\text {wall }}-T_{s}}{\Delta y_{s}},
$$

where $g$ indicates the gaseous and $s$ the solid phase. A cell-centered finitevolume discretization is used both for the gas and the solid phase. Thus $T_{g}$ and $T_{s}$ are cell center temperatures of the first fluid and solid volume, and $\Delta y_{g}$ and $\Delta y_{s}$ are the corresponding distances to the wall, respectively. This approach requires a good spatial resolution of the viscous sublayer and thus very fine grids close to solid walls. Moreover, $\lambda_{g}, T_{g}$, and $\Delta y_{g}$ have to be transferred from the gas to the solid phase solver. Since the computational grids do not match at the gas/solid interfaces, these values are interpolated between both grids to the positions where they are required. In the opposite direction, $T_{w}$ is returned to the gas phase solver and again, has to be interpolated to the correct gas phase position. This data exchange is performed in the same way from the external and the internal flows towards the solid strut.

Due to the second order central discretization chosen for the second order temperature derivative of Eq. (16), a septa-diagonal set of equations has to be solved for the solid in 3D. This is done with the in-house code HeatEQ [13], which is based on the Douglas-Gunn Alternating Direction Implicit (ADI) method [45]. This three-step algorithm is unconditionally stable and second order accurate in space and time. The resulting tri-diagonal sets of equations are solved efficiently by a Thomas algorithm [46]. HeatEQ uses structured multi-block grids, it is vectorized and parallelized and has been validated against analytical solutions as well as experimental test cases.

\section{Test case and numerical set up}

\subsection{Strut and channel geometry}

The simulated channel segment with lobed strut injector is plotted in Fig. 2. The channel has a constant cross section with a height of $38 \mathrm{~mm}$ 


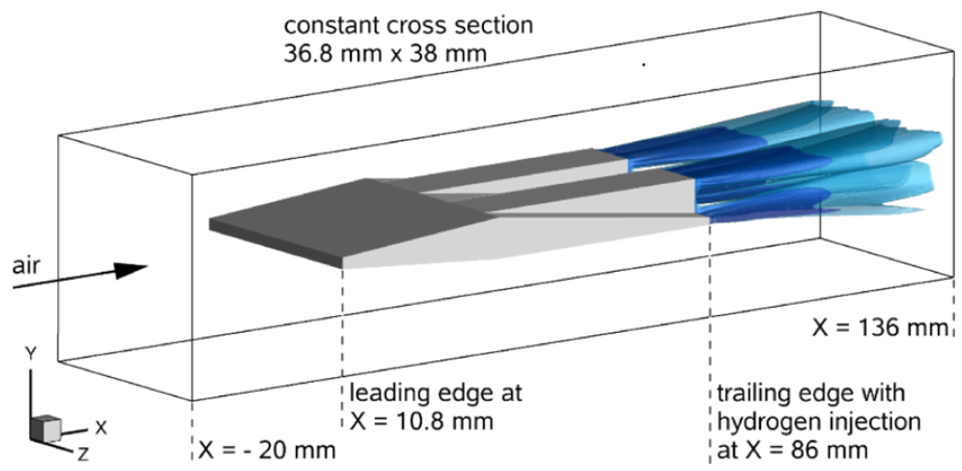

Figure 2: Simulated channel segment with lobed strut injector and two iso-surfaces (in blue) of constant hydrogen mass fractions.

and $36.8 \mathrm{~mm}$ depth. Both channel and strut geometry are according to the dimensions of the ITLR ground test facility at the University of Stuttgart $[23,16]$. In previous investigations [33], the strut injector had a sharp tip, as shown in the upper left sketch of Fig. 3. The origin of the channel coordinate system is at the front end of the sharp tip strut on the channel axis. In this paper, two blunt front geometries for the strut are investigated (lower two pictures of Fig. 3), which differ in the wall thickness of the vertical front part $(\Delta x=2.4 \mathrm{~mm}$ and $0.9 \mathrm{~mm})$. A reduction of the wall thickness enables better cooling. Due to the tip cutting, the length of the original strut ( 86 $\mathrm{mm}$ ) reduces to $75.2 \mathrm{~mm}$ and $73.7 \mathrm{~mm}$ and the front end positions are at $x=10.8 \mathrm{~mm}$ and $12.3 \mathrm{~mm}$, respectively. All simulations start at $x=-20$ $\mathrm{mm}$ to cover possible upstream effects. In case of the blunt front injector of Fig. 2 (2.4 mm front wall thickness), the inflow is $30.8 \mathrm{~mm}$ upstream of the injector. The strut is mounted at the channel side walls, allowing heat transfer towards the walls. All struts end at $x=86 \mathrm{~mm}$ where hydrogen is injected, but the simulated flow fields still extend $50 \mathrm{~mm}$ downstream up to the $x=136 \mathrm{~mm}$ position. Non-reactive flows are simulated, but even in case of combustion [18] the flame is usually lifted and does not increase the thermal load on the strut. Moreover, an upstream movement of the flame along the outer strut surface must be avoided because it can induce thermal choking.

Figure 4 shows a sketch of the investigated lobed strut injector. Hydrogen is injected (at supersonic speed) through a nozzle at the blunt end of the strut. As explained before, first investigations (also in experiment) used a sharp tip for the strut $[18,12,10]$. At real flight conditions, however, such a tip would 


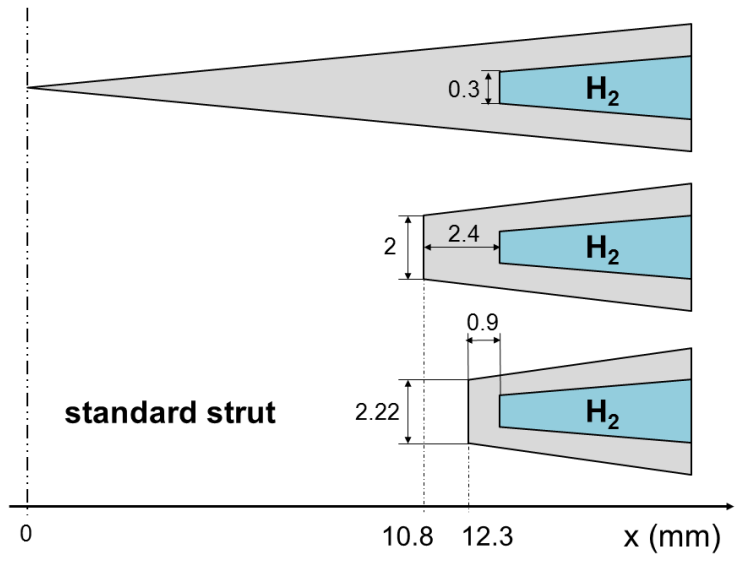

Figure 3: Investigated tips for the lobed strut injector, sizes in $\mathrm{mm}$. Sketches are not drawn to scale.

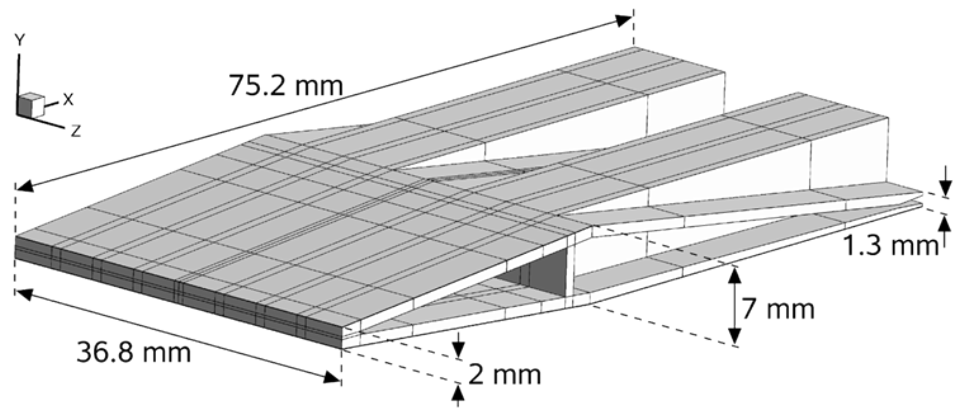

Figure 4: Geometry of the strut with $2.4 \mathrm{~mm}$ thickness of the blunt front, block structure, and lengths, sizes in $\mathrm{mm}$.

burn down because it cannot be cooled efficiently. At laboratory conditions with total temperatures between $1300 \mathrm{~K}$ and $1400 \mathrm{~K}$, a copper strut with sharp tip worked well, even at continuous operation [10]. The maximum temperatures at the tip are around $900 \mathrm{~K}$. At Mach 8 flight conditions, there are few materials (if any) able to withstand the high thermal load without an efficient active cooling. For this reason, the investigated struts have a blunt front $[12,13]$ which allows the internal coolant (hydrogen) to come close to the tip (see Fig. 3). One disadvantage of the blunt front is an associated small detached normal shock wave; this does, however, pass over quickly into an oblique shock. As will be shown later, the blunt front copper strut at 
Mach 8 flight conditions (total temperature of $3250 \mathrm{~K}$ ) and $\Phi=0.72$ has a lower maximum temperature (approximately $860 \mathrm{~K}$ ) than the sharp tip strut at $1300 \mathrm{~K}$ total temperature.

Hydrogen is fed to the strut through the side walls by two rectangular channels, as indicated by arrows in Fig. 5. All sizes of the lobed end are given in the lower sketch. Figure 5 additionally shows the hydrogen flow in-
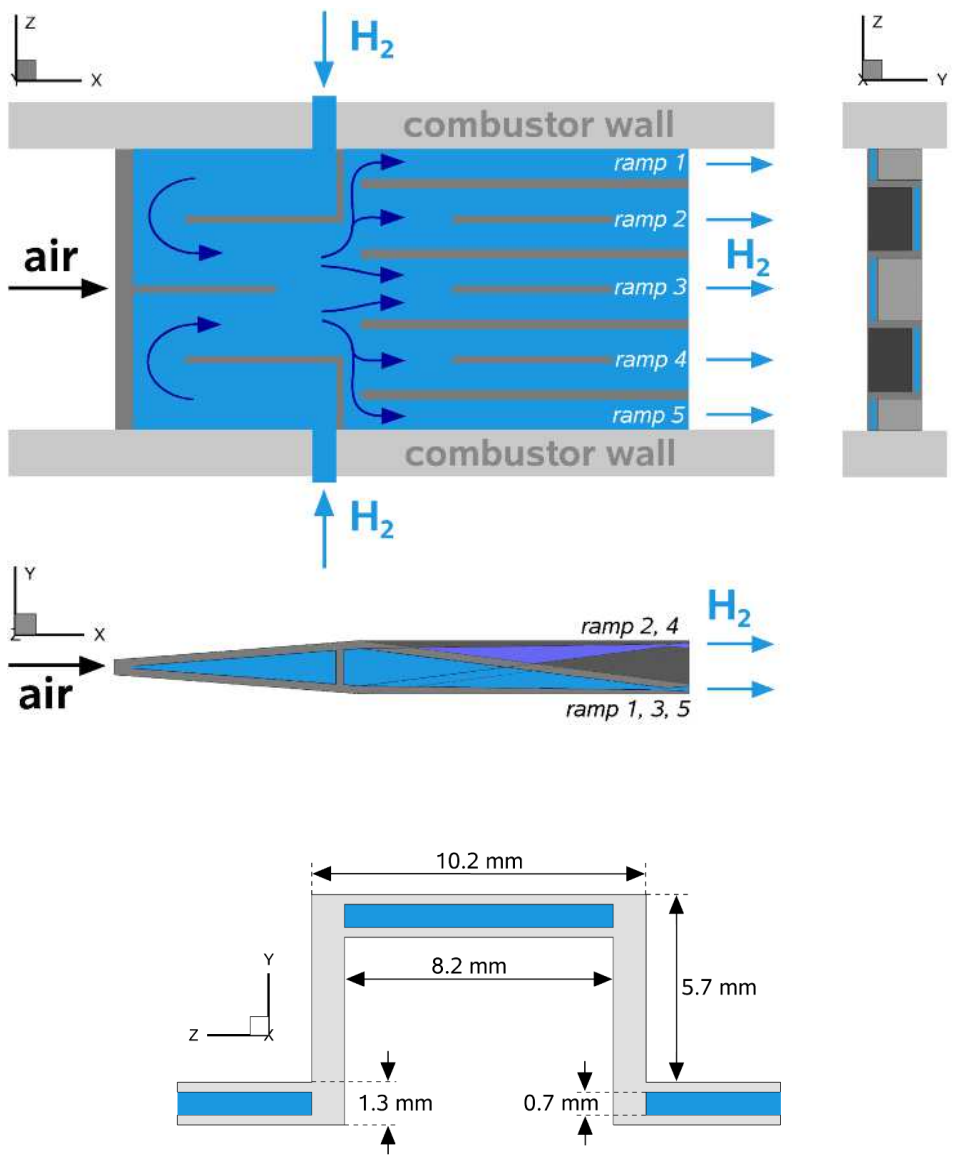

Figure 5: Interior structure of the strut and flow arrangement inside the strut (upper figures) with views from top, the side (below), and behind (right). Extension of the blunt end (bottom) and sizes. Hydrogen is depicted in blue.

side the strut. Hydrogen is first directed towards the tip in order to achieve good cooling of the highly thermally loaded front part. At the tip, the flow direction is changed and on its way to the nozzle, the hydrogen flux is split over the different ramps before injection. Nozzles inside the strut accelerate 
the hydrogen up to a Mach number of approximately 2.3 at the nozzle exit. The thickness of the internal walls needed to force the hydrogen flow in the desired directions is $1 \mathrm{~mm}$. This corresponds to the thickness of the upper and lower walls. The internal walls at the rear part of the strut are needed to distribute the hydrogen uniformly over the different ramps. Due to the limited depth of the strut $(18.4 \mathrm{~mm}$ from the combustor wall to the middle axis), the side walls noticeably affect the temperature distribution. However, the greatest part of the heat is taken by the strut and is transferred to the hydrogen and is thus used regeneratively. Moreover, the heated hydrogen reduces the ignition delay time and therefore contributes, at critical conditions, to flame stabilization.

\subsection{Computational grid, numerical set-up, and coupling}

Due to the complex geometrical structure of the strut injector, its computational grid requires a large number of blocks for discretization. Besides the lobed structure at the end of the strut a number of internal walls increase the complexity of the structured grid for the solid. Figure 6 shows the computational grids for the external channel flow (top), the internal hydrogen flow (middle), and for the strut material (bottom). In order to reduce the computational cost, only one half of the channel/strut is simulated, which is sufficient for steady-state RANS simulations. Symmetry boundary conditions are assumed at the channel middle plane. Due to the requirements of the $q-\omega$ low-Reynolds number turbulence model and to accurately resolve the heat fluxes at the walls, very fine grids $\left(y^{+} \approx 1\right)$ are used for all near-wall regions. The mesh in the solid is also strongly refined at all solid/fluid interfaces. In total, the grid consists of 463 blocks and 6.86 million volumes. The grid for the strut is divided into 327 blocks and has 0.42 million volumes. The grid for the internal flow has 54 blocks and 1.57 million volumes, and the grid for the external flow consists of 82 blocks and 4.88 million volumes. If required for parallelization, these blocks are further divided according to the number of available CPUs.

All simulations presented in this paper use identical air inflow conditions at the left side of the combustion chamber (see Fig. 2), which correspond to Mach 8 flight conditions. Due to the supersonic speed, all inflow values are fixed. Hydrogen enters the strut through a fuel supply tube at subsonic speed. Here, a mass flux boundary condition is employed to achieve the desired fuel mass flux [47]. The inflow conditions for air and hydrogen are summarized in Table 1. Depending on the chosen inlet pressure for hydrogen, 


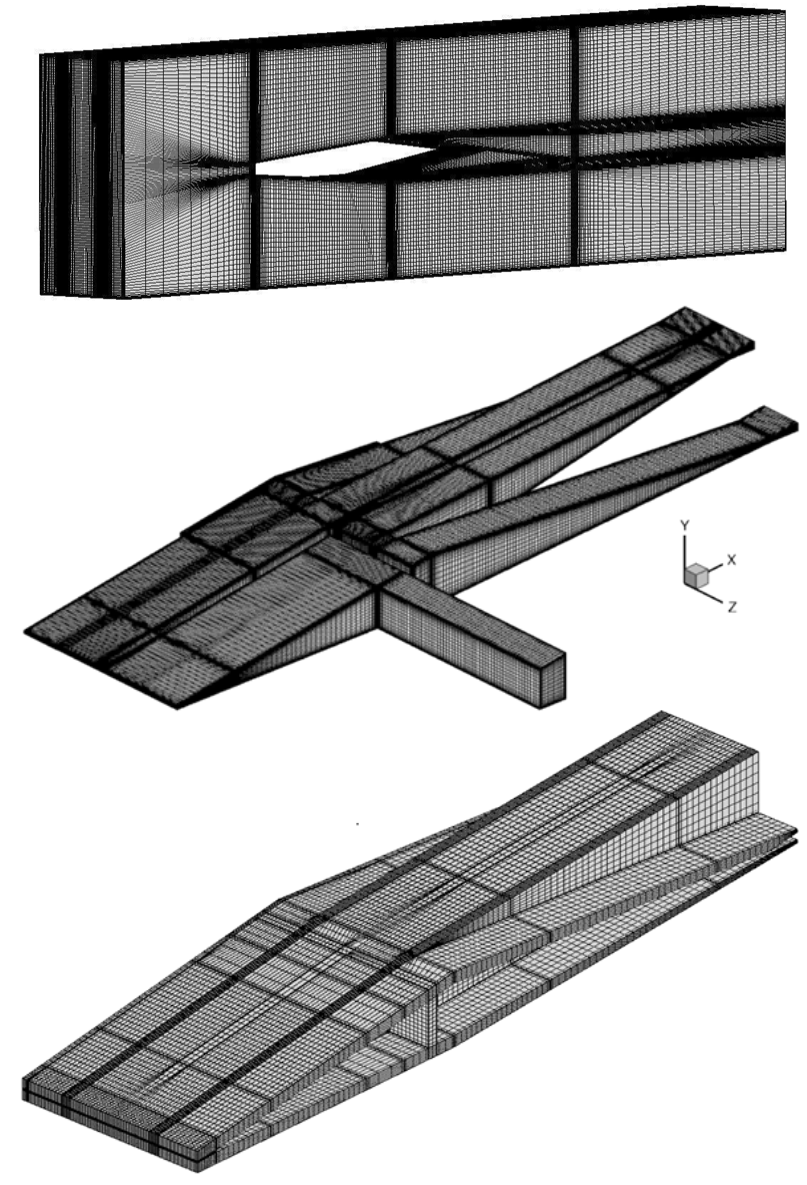

Figure 6: Computational grids for the external channel flow (top), the strut internal flow (middle), and the heat conduction simulation in the strut material (bottom).

three equivalence ratios $(\Phi=0.18,0.36$, and 0.72$)$ are realized. In all cases, a constant wall temperature of $T_{w}=450 \mathrm{~K}$ is set at the combustion chamber walls as well as at the walls of the hydrogen supply tubes, which cross the side walls.

For the steady-state results presented later, the internal and external flow fields as well as the temperature field of the strut are calculated separately. After a specified number of iterations, boundary data $\left(\lambda_{g}, T_{g}, \Delta y_{g}\right.$ and $T_{w}$, see Sect. 2) are exchanged. Between 15 and 20 such cycles are required to achieve a fully converged solution. However, this is possible only if an extrapolation of the exchanged data is performed [7]. Otherwise, nearly 
Table 1: Inflow conditions for air (combustion chamber) and hydrogen (strut injector). The hydrogen inlet pressures result in equivalence ratios of $\Phi=0.18,0.36$, and 0.72 .

\begin{tabular}{lll}
\hline & air & hydrogen \\
\hline Mach number (-) & 3.0 & 0.13 \\
static temperature (K) & 1160 & 290 \\
total temperature (K) & 3250 & 291 \\
velocity (m/s) & 2003 & 175 \\
static pressure (bar) & 0.97 & $12.0 / 24.0 / 48.0$ \\
\hline
\end{tabular}

twice as many couplings and iterations are needed. For every coupling cycle approximately 10,000 iterations are performed for the internal flow, 30,000 for the external flow, and 90,000 for the strut material, respectively. This is because of the strongly different temporal behaviors of the supersonic and subsonic flows on one side, and the heat conduction in the solid on the other side. Since heat conduction is the slowest process, couplings are performed until the change in maximum strut temperature is lower than $1 \mathrm{~K}$ per cycle.

\section{Results and discussion}

In order to get an overview of the external flow field, Figs. 7 and 8 show pressure, axial velocity, temperature, and turbulence variable $(q=\sqrt{k})$ contour plots for the simulated channel segment. The corresponding Mach number distribution is given in Fig. 9 (top). The figures are for the standard strut geometry (see Fig. 3), an equivalence ratio of $\Phi=0.18$, and $\mathrm{SiC}$ (silicone carbide) as strut material. However, the external flow fields using other hydrogen mass fluxes, materials, and front wall thicknesses mainly differ close to the wall. Thus, the given figures are representative for all simulations. All contour plots clearly show the shock wave in front of the strut which is reflected at the upper and lower channel walls. The reflected shocks hit the strut again approximately in the middle of the lobed strut structure. As will be shown later more clearly, this causes an increase in strut temperature. Thus, besides the high temperature zone at the tip, there is a second region where peak temperatures may occur. The bow shock is also responsible for a small subsonic zone upstream of the blunt front with pressures up to 10 bar and strong turbulence production (see $q$ contour plot). As a result, the flow field around the strut is completely turbulent. Downstream of the strut, the 


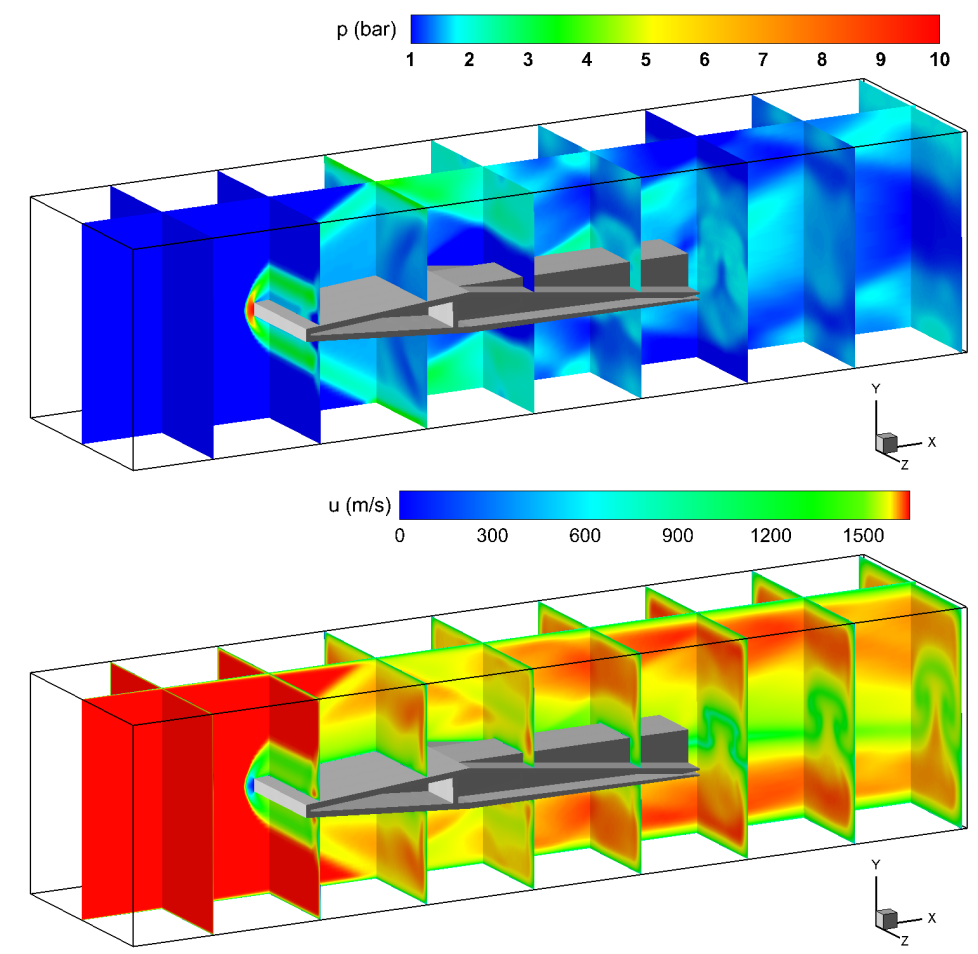

Figure 7: Flow fields around the standard $\mathrm{SiC}$ strut injector at $\Phi=0.18$, pressure (top), axial velocity (bottom).

lobed structure of the injected hydrogen becomes visible in the temperature, velocity, and $q$ distributions. An important point of the presented strut design is that the slender structure of the strut could be retained despite the blunt front. The size of the normal shock is kept small due to the low height of the front part of the strut, and its strength is limited by a quick transition into an oblique shock wave. Hence the high pressure (see Figs. 7 top) and high density region at the front is kept small. This is advantageous because a high density reduces the thermal diffusivity (see Eq. (17)) and increases the material temperature at the tip, which should be avoided as far as possible.

\subsection{Influence of the front wall thickness of the strut}

As described in Sect. 5.1, blunt fronts for the strut with wall thicknesses of $\Delta x=0.9 \mathrm{~mm}$ and $2.4 \mathrm{~mm}$ are investigated. Mach number distributions for both geometries are given in Fig. 9. The impact of $\Delta x$ on the front shock and the external flow field is minor and hardly visible. However, the strut tem- 


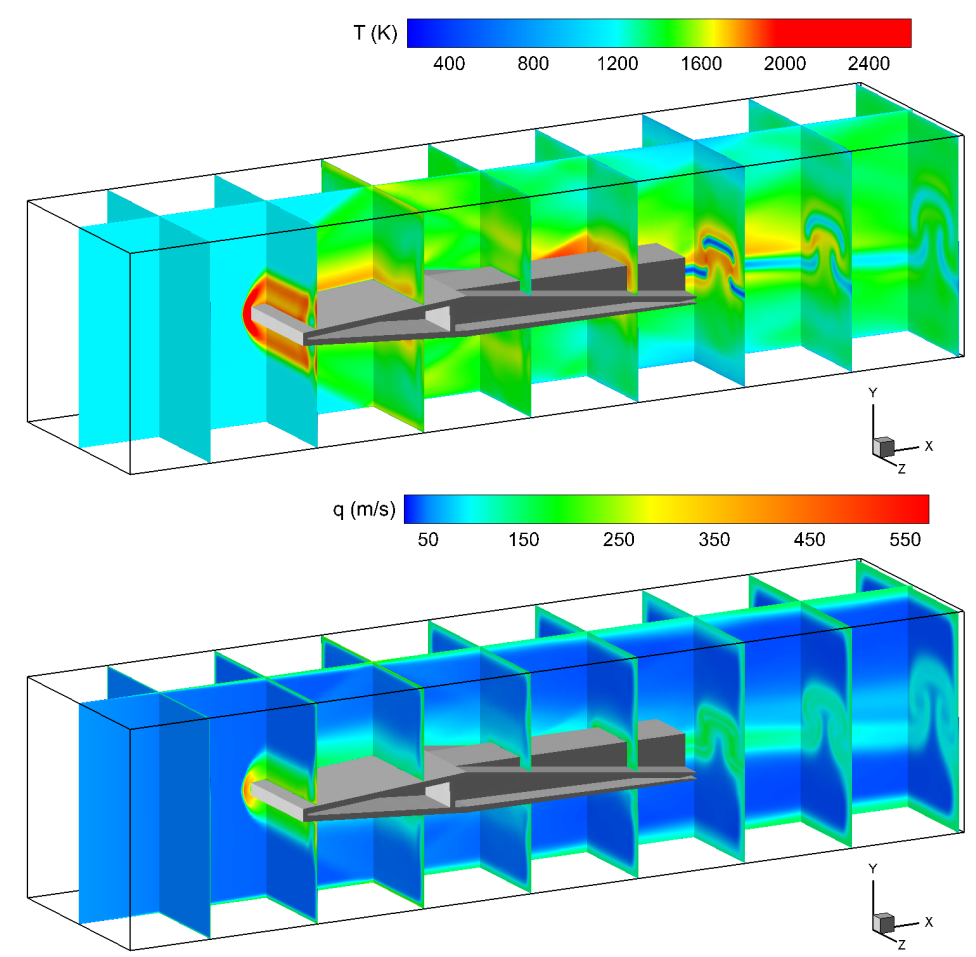

Figure 8: Flow fields around the standard $\mathrm{SiC}$ strut injector at $\Phi=0.18$, temperature (top), turbulence variable $q$ (bottom).

peratures differ significantly (strut temperatures are plotted in black/white in Fig. 9). This is shown more clearly in a second example in Fig. 10. The strut on the left side has $0.9 \mathrm{~mm}$ front wall thickness, the right one $2.4 \mathrm{~mm}$. The legends are different and related to the individual peak temperatures in order to highlight the effects at the front. In the rear part of the strut, both temperature distributions are quite similar. Results for copper struts at an equivalence ratio of 0.72 are given. However, the changes due to the described variation of $\Delta x$ are similar for other strut materials and hydrogen mass flow rates (see also Fig. 14). In the present case, the maximum temperature is $1004 \mathrm{~K}$ for the $\Delta x=2.4 \mathrm{~mm}$ strut, while $859 \mathrm{~K}$ is reached with $0.9 \mathrm{~mm}$ front wall thickness. This is a reduction of $145 \mathrm{~K}$ in peak temperature. For lower hydrogen mass flow rates $(\Phi=0.36$ and 0.18$)$, the drops for copper struts are $140 \mathrm{~K}$ and $179 \mathrm{~K}$, respectively. In case of the $\mathrm{SiC}$ struts from Fig. 9, the reduced front wall thickness causes a drop of $204 \mathrm{~K}$ in peak temperature (from $2050 \mathrm{~K}$ to $1846 \mathrm{~K}$ ), as can be seen from Fig. 14. Finally, Fig. 11 

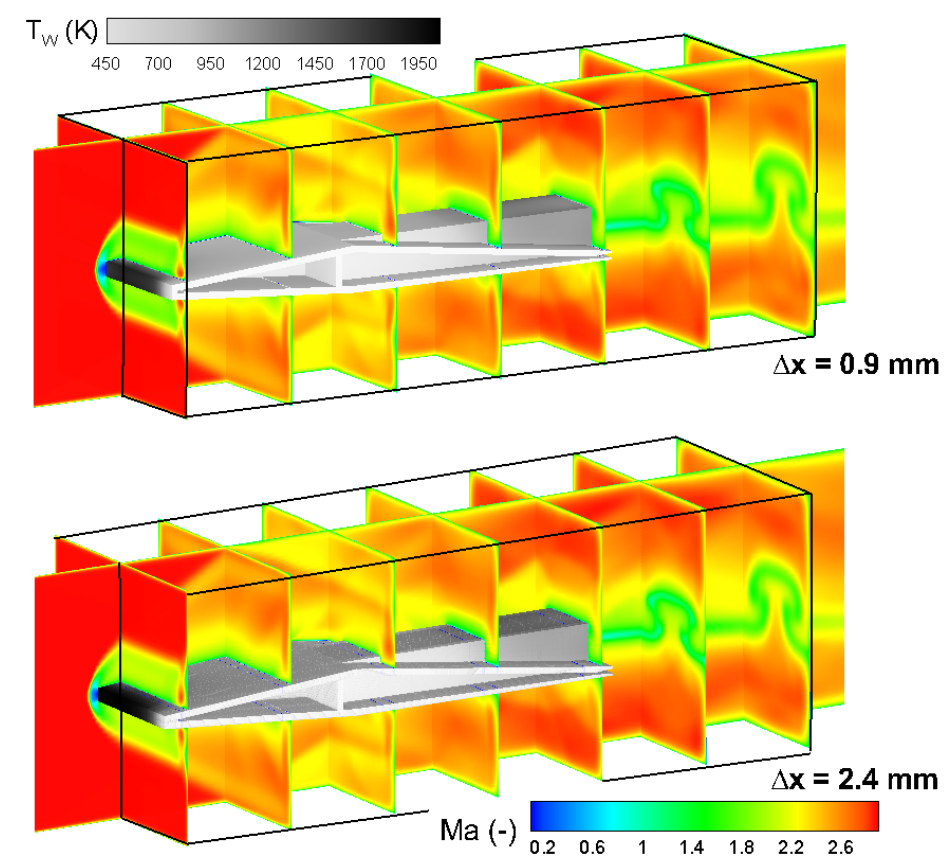

Figure 9: Mach number contours (color) and strut surface temperatures (black/white) of flow fields around $\mathrm{SiC}$ struts at $\Phi=0.18$ with $\Delta x=0.9 \mathrm{~mm}$ (top) and $\Delta x=2.4 \mathrm{~mm}$ (bottom) front wall thickness.

shows cuts at $z=1 \mathrm{~mm}$ ( $1 \mathrm{~mm}$ away from the middle plane) through the tips of the struts from Fig. 10. From these figures it becomes obvious that the increased wall thickness at the front is responsible for the higher peak temperature. Because of the low height of the cooling channel at the front end $(0.3 \mathrm{~mm})$, the tip of the strut is not cooled optimally. This becomes evident in the $\Delta x=2.4 \mathrm{~mm}$ strut case, but even the front of the $\Delta x=0.9$ $\mathrm{mm}$ strut could be cooled better. For that purpose, the strut length should be reduced still a little bit more, in order to increase the height of the internal hydrogen channel at the front from $0.3 \mathrm{~mm}$ up to approximately $1 \mathrm{~mm}$. Nevertheless, the smaller front wall thickness clearly outperforms the thicker one and the $\Delta x=0.9 \mathrm{~mm}$ configuration is defined to be the standard strut in the following investigations.

Due to the reflected shock waves, there is a second region in the rear part of the strut, where high material temperature may occur (see Fig. 10). Especially the vertical walls of the lobed structure (which are not internally cooled as the horizontal ones) show high temperatures towards the end. In the in- 


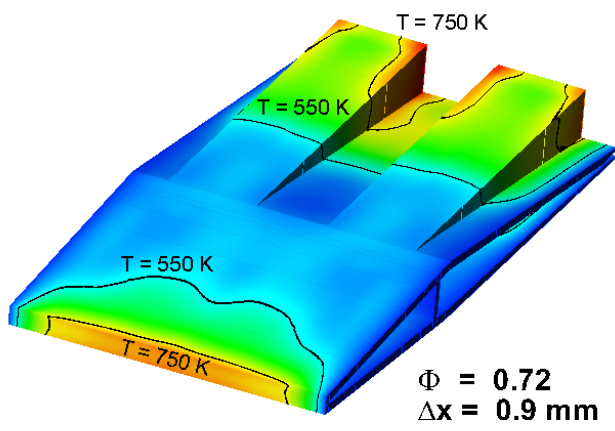

$\mathrm{T}(\mathrm{K})$

$460 \quad 520 \quad 580 \quad 640 \quad 700 \quad 760 \quad 820$

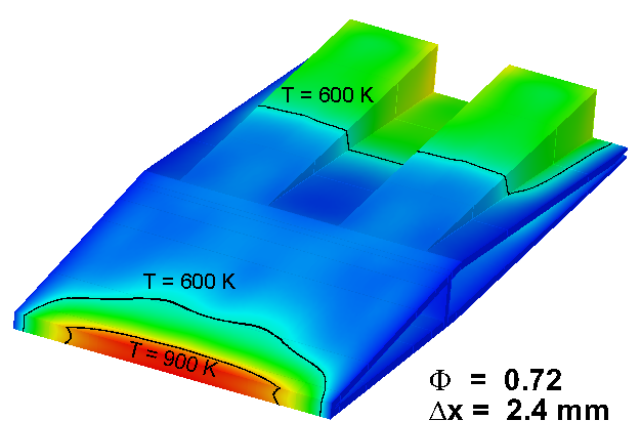

$\mathrm{T}(\mathrm{K})$

$\begin{array}{llllll}450 & 550 & 650 & 750 & 850 & 950\end{array}$

Figure 10: Simulated surface temperatures of lobed strut injectors with $0.9 \mathrm{~mm}$ (left) and $2.4 \mathrm{~mm}$ (right) front wall thickness (copper strut at $\Phi=0.72$ ).
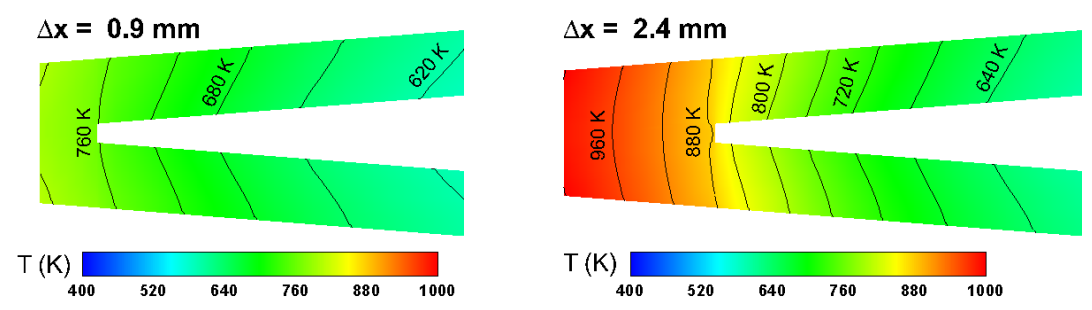

Figure 11: Simulated strut temperatures at the tips of struts with $0.9 \mathrm{~mm}$ (left) and 2.4 $\mathrm{mm}$ (right) front wall thickness (copper strut at $\Phi=0.72, \Delta T=40 \mathrm{~K}$, location of the cut is at $z=1 \mathrm{~mm})$.

vestigated cases this is not a serious problem, because at low equivalence ratios where the highest strut temperatures occur, the peak values are in the front. Otherwise struts as in $[33,18,15]$ could be used, where, in contrast to the present ones, hydrogen is injected through the vertical parts as well. In previous studies (e.g. [18]), the impact of different hydrogen injection areas along the lobed structure has been investigated. However, these investigations considered mixing efficiency and total pressure losses only while cooling was not taken into account. The result of these studies was that fuel injected along the vertical parts of the lobed structure does not efficiently mix with the surrounding air because it is injected directly or close to the center of the induced vortices. The present result points in the opposite direction and a compromise could be a strut (similar to [18]) where only parts of the vertical structure are open for fuel injection. This clearly shows that it is essential 
for strut design to take all aspects into account: mixing and combustion efficiency, total pressure losses, and cooling. Moreover, the cooling effect from the side walls is clearly visible in Fig. 10. This is the reason why the highest temperatures in the vertical walls are reached close to the channel axis.

\subsection{Influence of the equivalence ratio}

An important parameter for strut cooling is the mass flux of hydrogen injected through the strut; this mass flux should never fall below the required minimum value (for a given flight Mach number and strut). Typically the fuel mass flow is defined by combustion with the aim to achieve a desired thrust. The investigated combustion chamber is equipped with a single strut injector. This may be not sufficient for real configurations, where all or most of the incoming oxygen should be used for combustion. While centrally located strut injectors are well-suited to use the oxygen from the core flow, wall injectors would be able to additionally burn fuel with the oxygen, still available in the near wall regions. Examples for such staged scramjet combustion concepts are given in [48, 23]. As additional fuel may be added close to solid walls, equivalence ratios below $1(0.18,0.36$, and 0.72$)$ are used in the present strut injector study. The different equivalence ratios are realized by varying the inlet pressure at the fuel supply tubes while keeping inflow temperature and velocity constant (see Table 1). In this way, the hydrogen mass flow rate is changed while the air main flow is kept constant.

\subsubsection{Maximum temperatures}

Figure 12 shows hydrogen temperature (left side) and turbulence variable $q$ (right side) distributions inside the standard copper strut for three different equivalence ratios. The shown surfaces are always the middle planes located exactly in the middle between the upper and lower walls. In the first half of the strut this plane is at $y=0 \mathrm{~mm}$, while in the second half it is at $y \neq 0$ $\mathrm{mm}$ due to the ramps. Streamlines obtained from the velocity fields visualize flow structure and vortices. From picture to picture (top to bottom) the hydrogen mass flux is always doubled. As expected, this causes a reduction in hydrogen temperature. In case of the lowest equivalence ratio $(\Phi=0.18)$, the highest hydrogen temperatures of approximately $800 \mathrm{~K}$ appear in the small recirculation zone at the front close to the middle axis (at $x \approx 2 \mathrm{~mm}$, $z \approx 2 \mathrm{~mm}$ ) and downstream of the short internal walls in the middle of every $\operatorname{ramp}($ at $x \approx 75 \mathrm{~mm}, z \approx 0.5 \mathrm{~mm}$ and $9 \mathrm{~mm}$ ). These small high temperature regions disappear with increasing fuel mass flux. The short 

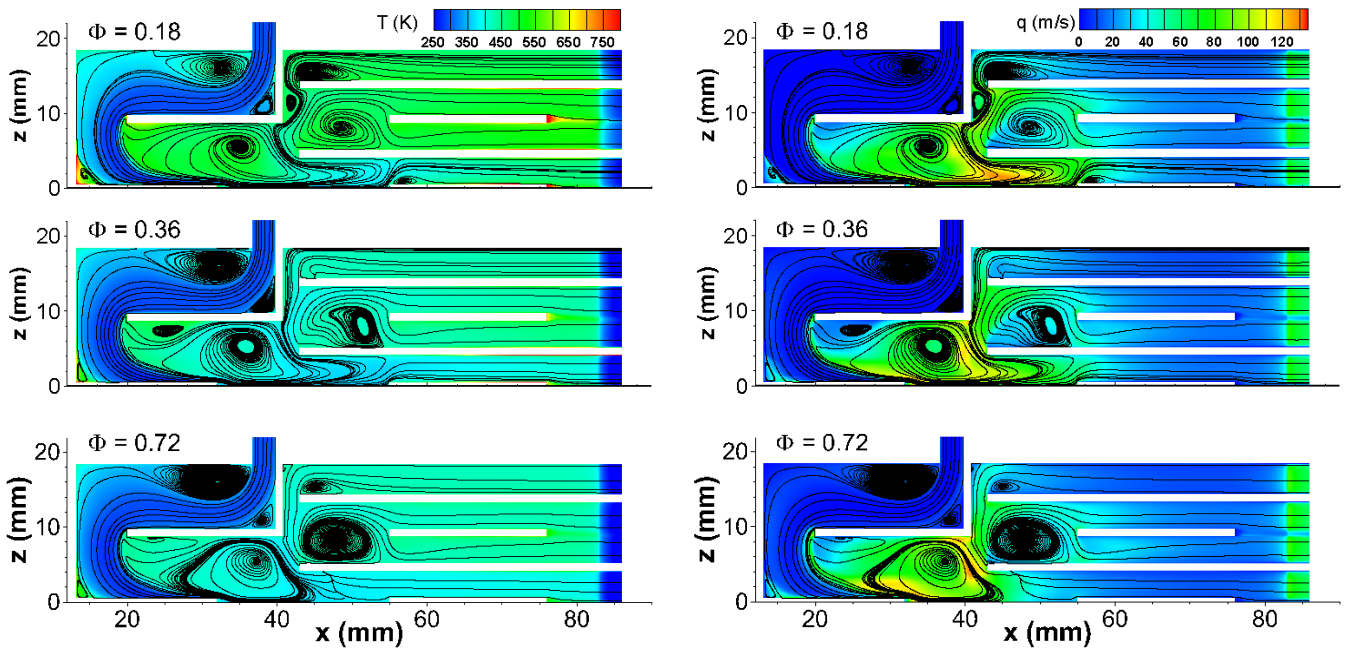

Figure 12: Temperature (left) and turbulence variable $q$ (right) distributions in the middle plane between the upper and lower strut walls. The figures are for one half of the strut and different equivalence ratios $(\Phi=0.18,0.36$, and 0.72 from top to bottom). Additionally streamlines of the velocity fields are given.

internal walls (at $55 \mathrm{~mm} \leq x \leq 76 \mathrm{~mm}$ and $y \approx 9 \mathrm{~mm}$ ) are used for flow homogenization. As can be seen from the streamlines, there are vortices upstream of these walls but there is a homogeneous flow field in the axial direction towards nozzle and outflow. Since the shown contour plots are in the middle between the upper and lower walls, the temperatures increase as these walls are approached. With increasing equivalence ratio, some of the smaller recirculation zones disappear and the flow is dominated by fewer, larger, and more stable vortices.

The $q=\sqrt{k}$ contour plots (right side of Fig. 12) show strong turbulence production after the flow direction changes towards the exit. Turbulence is produced in regions of strong velocity gradients. As described before, the different equivalence ratios are realized by changing the hydrogen inflow pressure. In this way the density is changed linearly, while the inflow velocity is kept constant. For these reasons, the differences in the velocity distributions (not shown) as well as in the $q$ contour plots (right side of Fig. 12) are relatively small. Differences in $q$ mainly occur due to the varying sizes and positions of the vortices formed inside the strut. The largest vortex is formed after the change in flow direction close to the middle axis. This vortex extends from $x \approx 20 \mathrm{~mm}$ up to $x \approx 55 \mathrm{~mm}$ in the uppermost figure and 


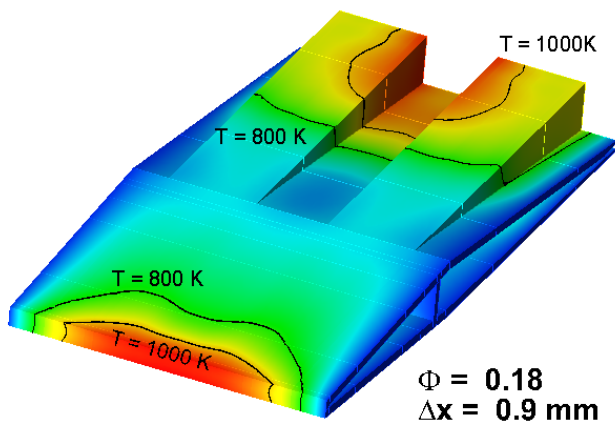

$\mathrm{T}(\mathrm{K})$

$450 \quad 550 \quad 650 \quad 750 \quad 850 \quad 950 \quad 1050$

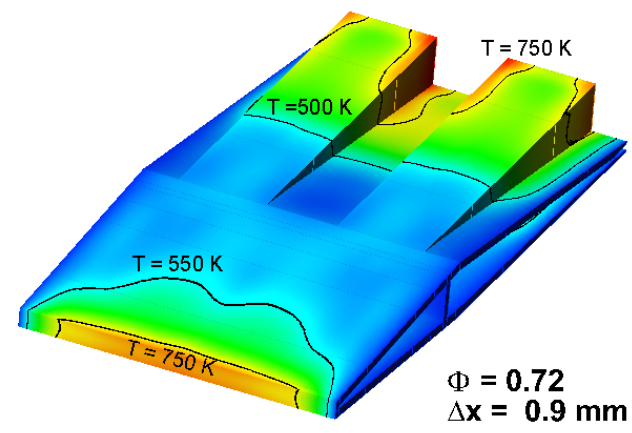

$\mathrm{T}(\mathrm{K})$

$\begin{array}{lllllll}460 & 520 & 580 & 640 & 700 & 760 & 820\end{array}$

Figure 13: Simulated surface temperatures of the standard copper strut injector at equivalence ratios of $\Phi=0.18$ (left side) and 0.72 (right side).

reduces in size (end is at $x \approx 51 \mathrm{~mm}$ and $45 \mathrm{~mm}$ for the middle and bottom figures, respectively) with increasing hydrogen mass flux. This changes the positions of turbulence peak values, too. For the lowest hydrogen mass flux, there is a region of high $q$ values in the ramp close to the middle axis $(x=$ $43 \mathrm{~mm}, y=2 \mathrm{~mm}$ ). This peak disappears with increasing mass flux and instead two peak regions appear further upstream at the right upper and left lower side of same vortex. The acceleration of the hydrogen in the nozzle (at $x \approx 83 \mathrm{~mm}$ ) causes a second increase in turbulence intensity shortly before injection.

The impact of an increased hydrogen mass flux on the strut surface temperature is illustrated in Fig. 13. Given are surface temperatures for the standard copper strut at equivalence ratios of 0.18 (left side) and 0.72 (right side). The peak temperatures at the front and at the end of the strut are significantly reduced by a higher hydrogen mass flow rate (note the different maximum values of both legends). This is shown quantitatively in Fig. 14, where the maximum strut temperatures are plotted over the equivalence ratio for different front wall thicknesses and two strut materials. In all cases, the peak temperatures significantly decrease (nearly linearly) with increasing hydrogen mass flux. For both the standard copper and silicone carbide strut the temperature reduction is approximately $280 \mathrm{~K}$ if $\Phi$ is increased from 0.18 to 0.72 . In the copper strut case, the maximum temperatures vary between $859 \mathrm{~K}$ and $1146 \mathrm{~K}$. This is in a range where, with some additional modifications identified in this paper, the strut may be used in practical applications. 


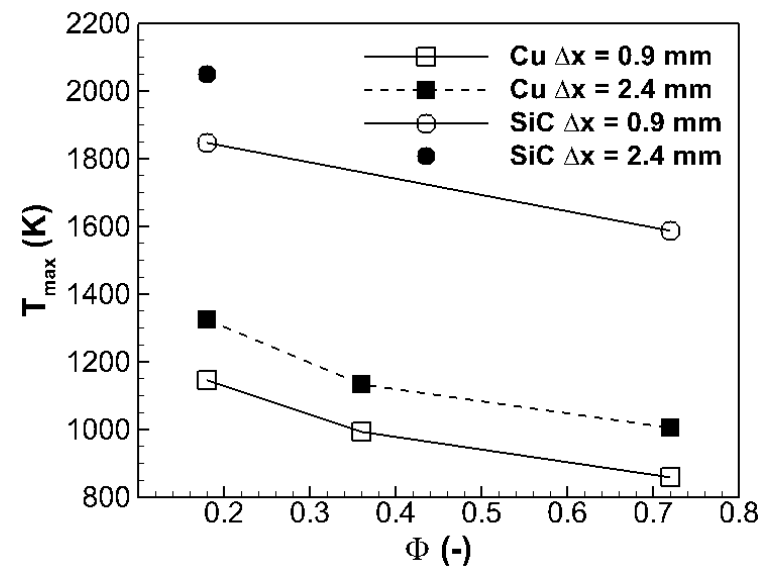

Figure 14: Strut material peak temperatures for copper and silicone carbide struts, two front wall thicknesses, and varying equivalence ratios.

In the silicone carbide case the maximum temperatures are nearly $700 \mathrm{~K}$ higher (from $1588 \mathrm{~K}$ up to $1846 \mathrm{~K}$ ). This could potentially also be within the range that advanced materials could withstand. Bouchez [30] reports about ceramic matric composite materials able to sustain $1850 \mathrm{~K}$ for several minutes in an oxidative environment. Tungsten (W) and molybdenum (Mo) are in between copper $(\mathrm{Cu})$ and silicone carbide $(\mathrm{SiC})$. However, much more information about a certain material is needed in order to qualify it for real scramjet application. This paper is a first step to identifying the temperature ranges and requirements for corresponding materials.

The increase in coolant mass flux also shifts the maximum temperatures from the front to the rear part of the strut. However, the differences between the front and rear maximum temperatures are relatively small. The highest temperatures at the end of the strut are at the non-cooled vertical walls of the lobed structure. This can be seen for the standard copper strut in Fig. 15, where a view from the back on the injection areas of the struts is given. For a better comparison, identical legends are used for the $\Phi=0.18$ (left side) and 0.72 (right side) cases. In the shown planes, the peak temperatures $(1116 \mathrm{~K}$ left side, $856 \mathrm{~K}$ right side) are always in the middle of the vertical wall, closest to the symmetry plane (indicated by dashed circles). The injected hydrogen is significantly heated inside the strut. Its distribution over the exit area is non-uniform with strong temperature differences between the boundary layer and the middle of the injected jet. This can be clearly seen in the left plot of 

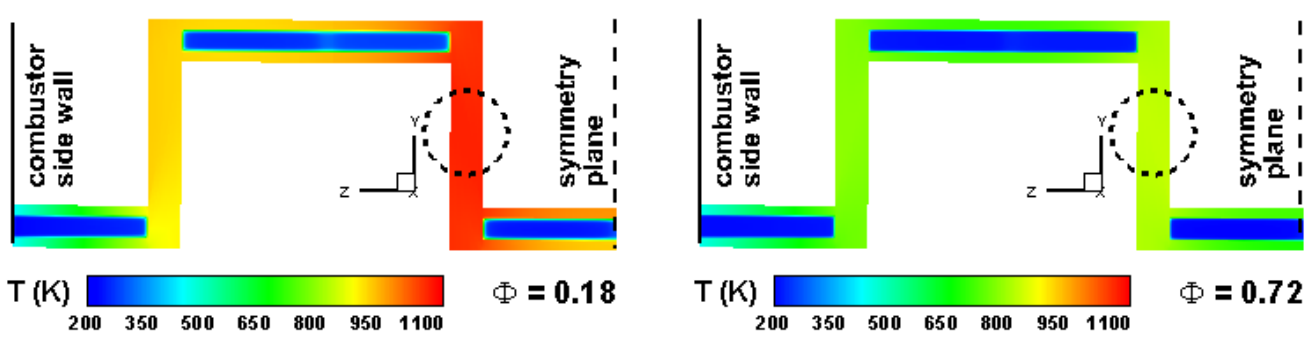

Figure 15: Surface temperature distributions of the strut materials and temperatures of the injected hydrogen at the strut ends, view from the back (standard copper strut). Regions of highest temperatures are indicated by dashed circles.

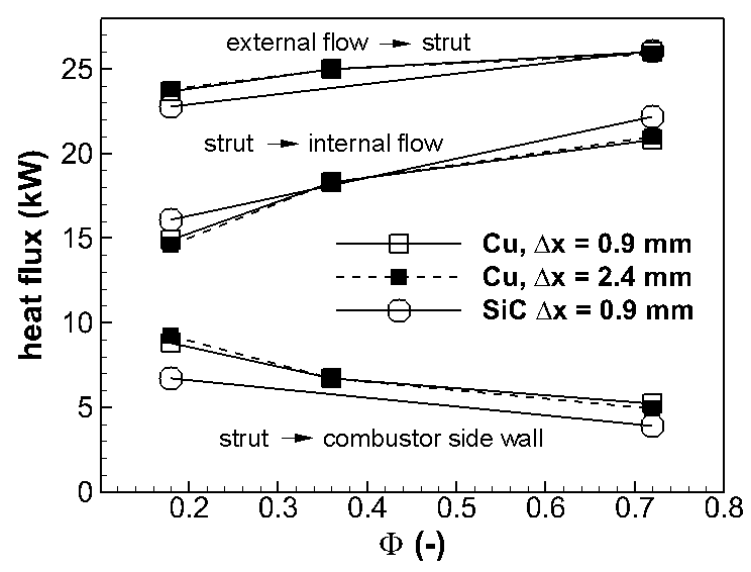

Figure 16: Heat fluxes for copper and silicone carbide struts with $0.9 \mathrm{~mm}$ and $2.4 \mathrm{~mm}$ (copper) front wall thickness at different equivalence ratios.

Fig. 15, where the hydrogen temperature reaches values of more than 1000 $\mathrm{K}$ directly at the wall, while it is quite cold $(285 \mathrm{~K})$ in the middle. Moreover, the distributions of the injected hydrogen also differ from ramp to ramp.

\subsubsection{Heat fluxes}

Heat fluxes (obtained by integration of the heat flux densities over the corresponding strut surfaces) from the external air flow towards the strut, from the strut to the internal hydrogen flow, and from the strut to the combustion chamber side walls are given in Fig. 16. Plotted are heat flux values for copper and silicone carbide struts at different equivalence ratios and front wall thicknesses. For the copper struts (and the other materials not shown 
in this diagram), the influence of the front wall thickness on the total heat fluxes is negligible. This is in contrast to the peak temperatures which are strongly influenced by $\Delta \mathrm{x}$. Figure 16 shows a rise in the heat fluxes from the air to the strut and from the strut to hydrogen if $\Phi$ is increased. At the same time, the heat fluxes to the side walls decrease. A four times higher hydrogen mass flux increases the heat flux to the strut by $8.8 \%$ only, but the heat flux from the strut to the hydrogen by $39.6 \%$. In case of the copper struts, between $63 \%(\Phi=0.18$ and $\Delta \mathrm{x}=0.9 \mathrm{~mm})$ and $81 \%(\Phi=0.72$ and $\Delta \mathrm{x}=$ $2.4 \mathrm{~mm}$ ) of the heat flux from the air to the strut is recovered by the injected hydrogen. The remaining part is transferred to the combustor side walls. The impact of different strut materials on the heat fluxes will be discussed in the next section. The heat flux $(25 \mathrm{~kW})$ from the external flow to the strut corresponds to a heat flux density of approximately $3.5 \mathrm{MW} / \mathrm{m}^{2}$. This is somewhat higher than the values obtained for the boundary layer flows in Fig. 1. Probable reasons are the detached normal shock wave at the blunt front and the shorter length of the strut, compared to the $1 \mathrm{~m}$ wall length taken in Fig. 1.

\subsection{Influence of the strut material properties}

Finally, different strut material properties (heat conductivities, specific heats, and densities) are investigated. The purpose here is to study the impact of material properties which roughly correspond to those of copper, molybdenum, tungsten, and silicon carbide. The strong disparity in strut peak temperature between copper and silicon carbide has already been shown in Fig. 14 for varying fuel mass fluxes.

Figure 17 shows temperature profiles for the thermal diffusivities of the materials mentioned above. Thermal diffusivity (see Eq. (17)) is a measure how fast heat diffuses in a given solid. For the present steady-state investigations, it is the most important parameter for the temperature distribution in the solid. While the data for copper is plotted up to $1250 \mathrm{~K}$ maximum temperature, the remaining properties are given up to $2000 \mathrm{~K}$, which may be higher than the practical usability of the materials. The references for the material property data as well as the material densities are summarized in Table 2. From these data, temperature dependent polynomials are formed and used in the simulations. For silicone carbide in particular, there is a large spectrum of varying grain sizes, porosities, and combinations with fiber materials. Accordingly the thermal conductivities differ strongly. The chosen 


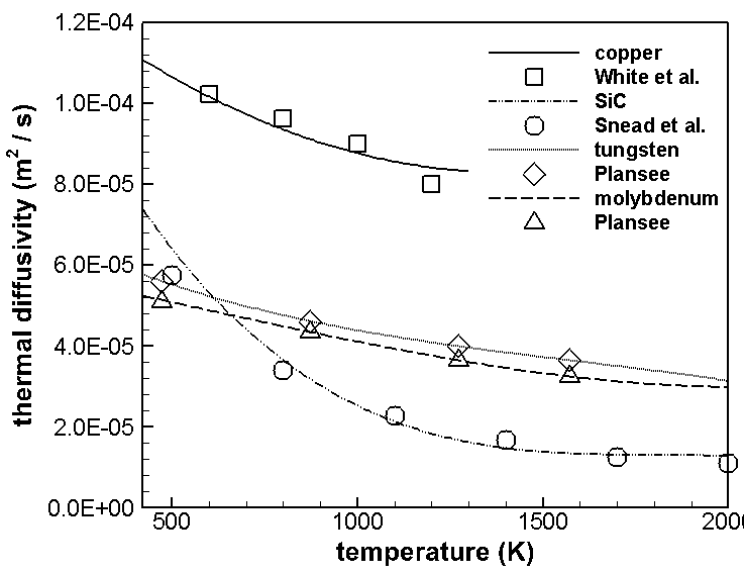

Figure 17: Thermal diffusivities for different materials, symbols: data from references, lines: polynomials used in the simulation.

Table 2: References for strut material data and densities used for the different materials.

\begin{tabular}{lllll}
\hline & $\mathrm{Cu}$ & Mo & W & SiC \\
\hline Ref. for $\lambda, c_{p}, \rho$ & {$[30],[31],[31]$} & all $[32]$ & all $[32]$ & all [33] \\
$\rho\left(\mathrm{kg} / \mathrm{m}^{3}\right)$ & $8.92 \cdot 10^{3}$ & $10.2 \cdot 10^{3}$ & $19.3 \cdot 10^{3}$ & $3.21 \cdot 10^{3}$ \\
\hline
\end{tabular}

$a_{\mathrm{SiC}}$ values in this paper likely are at the upper end of the spectrum. Molybdenum and tungsten have very similar heat conductivities and diffusivities despite their different densities. For all materials the thermal diffusivity reduces strongly with increasing temperature.

\subsubsection{Maximum temperatures}

The different strut materials are investigated for the worst conditions used in this paper, which are an equivalence ratio of 0.18 and $2.4 \mathrm{~mm}$ front wall thickness. This is in contrast to Sect. 6.2.1, where results for the better standard strut (copper and silicon carbide) have been given. Figure 18 shows surface temperatures for copper, molybdenum, and silicone carbide struts (from left to right). As the differences between tungsten and molybdenum are minor, only the latter is shown here. Identical legends are used for a better comparison. As expected, the peak temperatures increase from left to right due to the decreasing thermal diffusivity. The corresponding values 

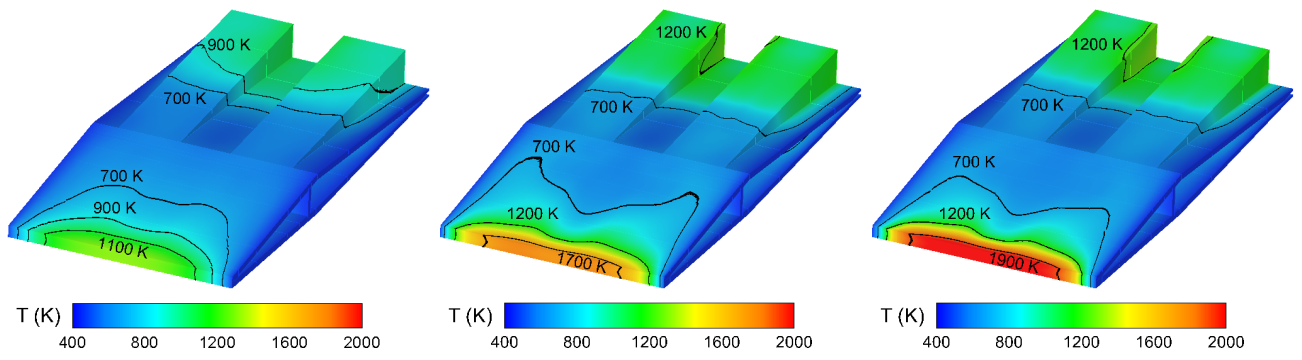

Figure 18: Calculated strut surface temperatures using different material properties: Copper, molybdenum, and silicone carbide from left to right.

Table 3: Peak temperatures of the $\Delta \mathrm{x}=2.4 \mathrm{~mm}$ strut at $\Phi=0.18$ for different strut materials (upper line) and heat fluxes from air to strut and strut to hydrogen (lower two lines) for the same strut and operating conditions.

\begin{tabular}{lllll}
\hline & $\mathrm{Cu}$ & $\mathrm{Mo}$ & $\mathrm{W}$ & $\mathrm{SiC}$ \\
\hline$T_{\max }(\mathrm{K})$ & 1325.3 & 1814.7 & 1795.1 & 2050.0 \\
$q_{\text {air-strut }}(\mathrm{kW})$ & 23.8 & 22.5 & 22.6 & 22.5 \\
$q_{\text {strut-H }}(\mathrm{kW})$ & 14.6 & 15.9 & 15.9 & 15.6 \\
\hline
\end{tabular}

are summarized in the first line of Table 3. In contrast to copper, the peak temperature of the silicone carbide strut is $725 \mathrm{~K}$ higher, while those of molybdenum and tungsten exceed the copper value by nearly $500 \mathrm{~K}$. Even in the case of the better cooled strut with $0.9 \mathrm{~mm}$ front wall thickness, the difference between copper and silicone carbide is still $700 \mathrm{~K}$, as can be seen in Fig. 14. Significant differences occur mainly at the tip of the strut as well as (somewhat smaller) in the rear part. In the middle part of the struts (see Fig. 18), where the wall thickness is nearly uniform and all walls are cooled well, the disparity due to the different materials becomes very small (in the region between the $700 \mathrm{~K}$ isolines).

Figure 19 shows cuts through the front parts of the standard copper (upper figure) and silicone carbide (lower figure) struts for $\Phi=0.72$, which confirm the observations above. The cuts are taken $2 \mathrm{~mm}$ away from the middle axis (at $z=2 \mathrm{~mm}$ ) in order to avoid the vertical wall at the axis. Identical legends are used. Note, however, that the legend for the SiC strut and the isolines end at $800 \mathrm{~K}$. While there are large differences at the tip, the temperature distributions at $x>17 \mathrm{~mm}$ become quite similar. This can 


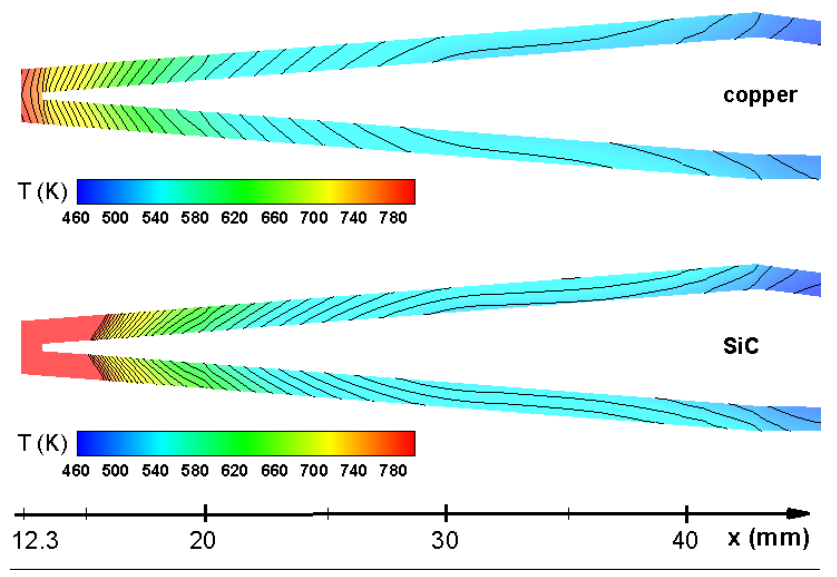

Figure 19: Cuts at $z=2 \mathrm{~mm}$ through the front parts of the standard copper (top) and silicone carbide (bottom) struts for $\Phi=0.72$ (isolines are plotted with $\Delta T=10 \mathrm{~K}$ ).

be seen on the left side of Fig. 20, too, where the outer and inner surface temperatures along the upper wall (at the interfaces from the solid to the outer air and to the inner hydrogen flow) are plotted for the cuts shown above. Between $x=25 \mathrm{~mm}$ and $x=45 \mathrm{~mm}$, the temperature profiles for copper and $\mathrm{SiC}$ at the strut/hydrogen interface are nearly identical. However, the temperatures at the air interfaces differ due to the different thermal diffusivities of the materials. To show this more clearly, the corresponding temperature differences $\Delta T_{W}$ over the wall thickness are plotted for the upper wall on the right side of Fig. 20. For $x>20 \mathrm{~mm}$, where the temperatures are below $600 \mathrm{~K}, \Delta T_{W}$ for the $\mathrm{SiC}$ strut is approximately two times higher than for the copper strut. This corresponds pretty well to the ratio of the thermal diffusivities at this temperature.

It can thus be summarized that the different strut materials cause strongly varying peak temperatures at the front. Due to the uncooled vertical walls of the lobed structure, there is a second high temperature region at the end, which again is influenced by material properties. In between a large part of the strut exists, where the surface temperatures are hardly influenced by the material. Here, the higher thermal diffusivity of copper causes a smaller $\Delta T_{W}$ over the wall thickness, however, its impact on the wall surface temperatures is negligible. Therefore an important point for practical applications should be to achieve a strut temperature distribution that is as homogeneous as possible. 

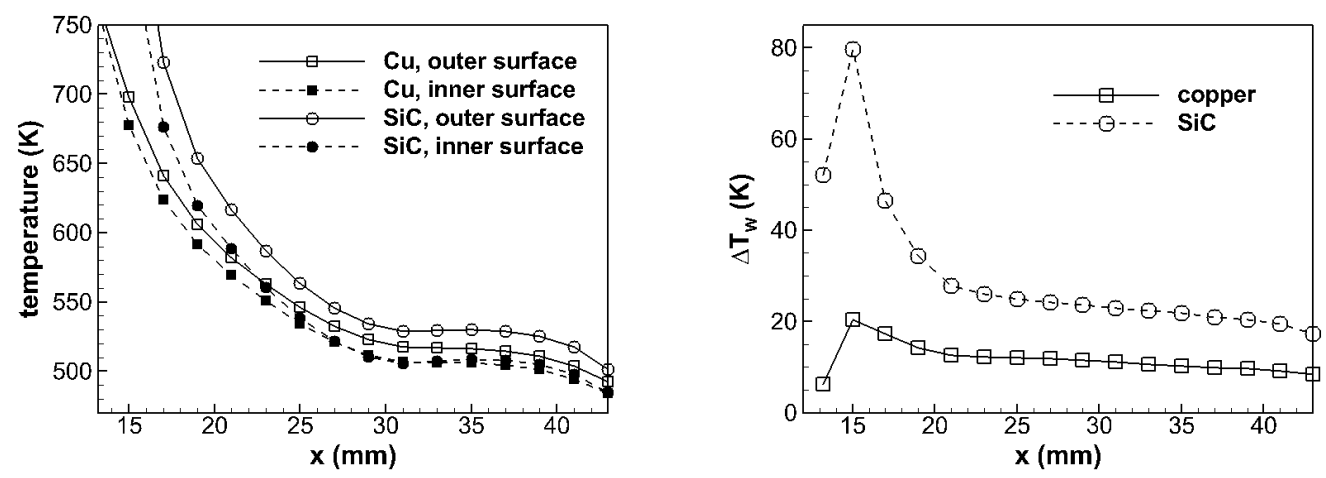

Figure 20: Temperature profiles (left side) along the outer and inner strut surface of the upper wall (see Fig. 19) and corresponding temperature differences (right side) for copper and $\mathrm{SiC}$ struts.

\subsubsection{Heat fluxes}

The heat fluxes (obtained by integration over the corresponding strut surface) from the external flow to the strut as well as from the strut to the hydrogen are quite similar for the different materials. This has been as shown in Fig. 16 for standard copper and silicon carbide struts. In the second and third line of Table 3, the wall heat fluxes are summarized for the $\Delta \mathrm{x}=2.4$ $\mathrm{mm}$ strut at $\Phi=0.18$ for all investigated materials. The differences in wall heat flux stemming from material properties are smaller than $10 \%$.

The presented results show that the impact of thermal diffusivity is important first of all at locations which are not efficiently cooled. On the other hand, the heat flux to the coolant is dominated by the outer surface temperature of the strut, as demonstrated in Fig. 1 for a flat plate. Because the available cooling capacity is a critical factor, the effect of reduced heat flux at higher temperatures could be used, if the strut material is able to withstand it and if it is possible, to achieve a uniform temperature distribution. In this case the fuel could be used first to cool the combustor walls before it is fed to the strut. Due to the elevated temperature the heat fluxes towards the strut would reduce significantly. As the strut is always the last part to be cooled before fuel injection, it will automatically be exposed to the highest temperatures. It therefore would be extremely useful to have a strut material which is able to withstand such temperatures and additionally offers a high thermal conductivity and diffusivity. 


\subsection{Summary and conclusions}

In the presented numerical study, small but important modifications of the front part of a lobed strut injector have been investigated. Moreover, different fuel mass fluxes and strut materials have been studied. All simulations are for Mach 8 flight conditions. Important results of the study are:

1. A blunt front for the strut should be used at real flight conditions.

2. Peak temperatures in the rear part of the strut can be avoided, if fuel is injected in the vertical parts, too.

3. As the degree of cooling has a strong influence on fuel injection temperature, pressure, and velocity, it subsequently affects ignition delay, mixing, and combustion.

4. It is important to keep the strut temperature as homogeneous as possible.

5. With the described minor modifications, the lobed copper strut injector should be able to work well over a wide range of fuel mass fluxes.

6. The impact of different material properties on peak temperatures and heat fluxes is identified.

In comparison to many other strut injector concepts, the present one not only achieves very good mixing (as shown before in $[12,18,19]$ ), but it also can be cooled efficiently over a wide range of operating conditions. In general, cooling has to be considered alongside fluid mechanical considerations during the design of a scramjet fuel injector.

\section{Acknowledgement}

This work has been performed within the GRK 1095/2 project "AeroThermodynamic Design of a Scramjet Engine for Future Space Transportation Systems". It has been supported by the Deutsche Forschungsgemeinschaft (DFG). We gratefully acknowledge the funding by the DFG and thank the High Performance Computing Center Stuttgart (HLRS) for providing computational resources.

\section{References}

[1] J. M. Donohue, Dual-mode scramjet flameholding operability measurements, Journal of Propulsion and Power 30 (2014) 592-603. 
[2] C. Aguilera, K. H. Yu, Supersonic mixing enhancement using fin-guided fuel injection, Journal of Propulsion and Power 31 (2015) 1522-1543.

[3] T. Kanda, G. Masuya, F. Ono, Y. Wakamatsu, Effect of film cooling/regenerative cooling on scramjet engine performance, Journal of Propulsion and Power 10 (1994) 613-624.

[4] J. Qin, W. Zhou, W. Bao, D. Yu, Thermodynamic optimization for a scramjet re-cooled cycle, Acta Astronautica 66 (2010) 1449-1457.

[5] K. D. Song, S. H. Choi, S. Scotti, Transpiration cooling experiment for scramjet engine combustion chamber by high heat fluxes, Journal of Propulsion and Power 22 (2006) 96-1ß2.

[6] T. Langener, J. von Wolfersdorf, J. Steelant, Experimental investigation on transpiration cooling for scramjet application using different coolants, AIAA Journal 49 (2011) 1409-1419.

[7] H. Huang, D. R. Sobel, L. J. Spadaccini, Endothermic heat-sink of hydrocarbon fuels for scramjet cooling, AIAA paper 2002-3871 (Aug. 2002).

[8] D. Zhang, Y. Feng, S. Zhang, J. Qin, K. Cheng, W. Bao, D. Yu, Quasione-dimensional model of scramjet combustor coulped with regenerative cooling, Journal of Propulsion and Power 32 (2016) 687-697.

[9] D. Yan, G. He, W. Li, D. Zhang, J. Qin, Thermal analysis of regenerative-colled pylon in multi-mode rocket based combined cycle engine, Acta Astronautica 148 (2018) 121-131.

[10] E. Daniau, M. Bouchez, R. Bounaceur, F. Battin-Leclerc, P. N. Marquaire, R. Fournet, Contribution to scramjet active cooling analysis using n-dodecane decomposition model as a generic endothermic fuel, AIAA paper 2003-6920 (2003).

[11] D. Zhang, J. Qin, K. Xie, Y. Feng, W. Bao, Thermal behavior inside scramjet cooling channels at different channel aspect ratios, Journal of Propulsion and Power 32 (2016) 57-69.

[12] P. Gerlinger, Y. H. Simsont, Numerical simulation of the internal and external flow fields and heat fluxes of a lobed strut injector, ISABE 2013-1627 (2013). 
[13] Y. H. Simsont, P. Gerlinger, High order numerical simulation of the thermal load on a lobed strut injector for scramjet application, International Journal for Numerical Metods in Fluids 82 (2016) 417-436.

[14] V. Vergine, C. Ground, L. Maddalena, Strut injectors for scramjets: Total pressure losses in two streamwise vortex interactions, Journal of Propulsion and Power 33 (2017) 1140-1150.

[15] Y. H. Simsont, P. Gerlinger, Numerical investigation of a complete scramjet model with central strut injection, ISABE paper 2013-1619 (2013).

[16] N. Dröske, Förste, B. Weigand, J. von Wolfersdorf, Thermal investigation of an internally cooled strut injector for scramjet application at moderate and hot gas conditions, Acta Astronautica 132 (2017) 177-191.

[17] K. Srinivasan, P. K. Maurya, K. Abhishek, S. L. N. Desikan, B. Murugan, Supersonic combustion of a scramjet engine using hydrogen fuel in a shock tunnel, AIAA Journal 56 (2018) 3600-3609.

[18] P. Gerlinger, P. Stoll, M. Kindler, F. Schneider, M. Aigner, Numerical investigation of mixing and combustion enhancement in supersonic combustors by strut induced streamwise vorticity, Aerospace Science and Technology 12 (2008) 159-168.

[19] S. Zhu, X. Xu, P. Ji, Flame stabilization and propagation in dual-mode sramjet with staged strut injectors, AIAA Journal 55 (2017) 171-179.

[20] P. Manna, R. Behera, D. Chharaborty, Liquid-fueled strut-based scramjet combustor design: A computational fluid dynamics approach, Journal of Propulsion and Power 24 (2008) 274-281.

[21] S. L. N. Desikan, J. Kurian, Strut-based gaseous injection into a supersonic stream, Journal of Propulsion and Power 22 (2006) 474-473.

[22] W. Bao, Q. Yang, J. Chang, Y. Zong, J. Hu, Dynamic characteristics of combustion mode transition in a strut-based scramjet combustor model, Journal of Propulsion and Power 29 (2013) 1244-1248.

[23] F. J. Förster, N. Dröske, M. N. Bühler, J. von Wolfersdorf, B. Weigand, Analysis of flame characteristics in a scramjet combustor with a staged 
fuel injection using common path focusing schlieren and flame visualization, Combustion and Flame 168 (2016) 204-215.

[24] S. Kumar, S. Das, S. Sheelam, Application of cfd and the kriging method for optimizing the performance of a generic scramjet combustor, Acta Astronautica 101 (2014) 111-119.

[25] S. Sujith, T. M. Murugamandam, J. Kurian, Effect of trailing ramp angle in strut-based injection in supersonic flow, Journal of Propulsion and Power 29 (2013) 66-78.

[26] O. A. Buchmann, Thermal-structural design study of an airframe integrated scramjet, NASA contractor report 3141 (1979).

[27] S.-Y. Ho, A. Paull, Coupled thermal, structural and vibrational analysis of a hyperbolic engine for flight test, Aerospace Science and Technology 10 (2006) 420-426.

[28] B. R. Capra, L. M. Brown, R. R. Boyce, S. C. Tirtey, Aerothermalstructural analysis of a rocket-launched mach 8 scramjet experiment: Ascent, Journal of Spacecraft and Rockets 52 (2015) 684-696.

[29] A. Purwar, D. R. Mahapatra, N. Thakor, K. S. Priyamvada, R. Mukherjee, A methodology for coupled thermal-structural analysis and structural design of scramjet combustor, in: G. Ben-Dor, O. Sadot, O. Igra (Eds.), 30th International Symposium on Shock Waves, Vol. 1, Springer, Cham, 2017, pp. 245-250.

[30] M. Bouchez, Scramjet thermal management, Lecture von Karman Institute rto-en-avt-185 (2010).

[31] S. J. Scotti, C. J. Martin, S. H. Lucas, Active cooling design for scramjet engines using optimization methods, AIAA paper 88-2265 (2010).

[32] D. Riggins, R. Tackett, T. Taylor, A. Auslender, Thermodynamic analysis of dual-mode scramjet engine operation and performance, AIAA paper 2006-8059 (2006).

[33] P. Gerlinger, P. Kasal, P. Stoll, D. Brüggemann, Experimental and theoretical investigation on $2 \mathrm{D}$ and $3 \mathrm{D}$ parallel hydrogen/air mixing in a supersonic flow, ISABE 2001-1019 (2001). 
[34] M. Kindler, P. Gerlinger, M. Aigner, Hybrid RANS/LES of lobed strut injectors in supersonic flow, ISABE 2013-1625 (2013).

[35] T. J. Coakley, P. G. Huang, Turbulence modeling for high speed flows, AIAA paper 92-0436 (1992).

[36] Y.-H. Choi, C. L. Merkle, The application of preconditioning in viscous flows, Journal of Computational Physics 105 (1993) 207-223.

[37] P. Gerlinger, P. Stoll, D. Brüggemann, An implicit multigrid method for the simulation of chemically reacting flows, Journal of Computational Physics 146 (1998) 322-345.

[38] P. Gerlinger, H. Möbus, D. Brüggemann, An implicit multigrid method for turbulent combustion, Journal of Computational Physics 167 (2001) $247-276$.

[39] P. Gerlinger, Multi-dimensional limiting for high-order schemes including turbulence and combustion, Journal of Computational Physics 231 (2012) 2199-2228.

[40] A. Jameson, S. Yoon, Lower-upper implicit schemes with multiple grids for the Euler equations, AIAA Journal 25 (7) (1987) 929-935.

[41] J. S. Shuen, Upwind differencing and LU factorization for chemical nonequilibrium Navier Stokes equations, Journal of Computational Physics 99 (1992) 233-250.

[42] M.-S. Liou, A sequel to AUSM, part ii: AUSM ${ }^{+}$-up for all speeds, Journal of Computational Physics 214 (2006) 137-170.

[43] P. Gerlinger, K. Nold, M. Aigner, Influence of reaction mechanisms, grid spacing, and inflow conditions on the numerical simulation of lifted supersonic flames, International Journal for Numerical Methods in Fluids 62 (2010) 1357-1380.

[44] V. Talyzin, V. Vlasenko, O. Voloschenko, M. Ivankin, M. Nikolaev, Experimental investigation of the hydrogen combustion chamber and its complement with 2.5d CFD data, HiSST paper 2018-1701068 (2018). 
[45] J. Douglas, J. E. Gunn, A general formulation of alternating direction methods - part i: Parabolic and hyperbolic problems, Journal of Numerical Mathematics 6 (1964) 428-453.

[46] D. A. Anderson, J. C. Tannehill, R. H. Pletcher, Computational Fluid Mechanics and Heat Transfer, Second Edition, McGraw Hill Higher Education, 1984.

[47] A. Jirásek, Mass flow boundary conditions for subsonic inflow and outflow boundary, AIAA Journal 44 (5) (2006) 939-947.

[48] S. Tomioka, K. Kobayashi, K. Kudo, A. Murakami, Distributed fuel injection for performance improvement of staged supersonic combustor, Journal of Propulsion and Power 21 (2005) 760-762. 